\title{
Constraining the origin and models of chemical enrichment in galaxy clusters using the Athena X-IFU
}

\author{
F. Mernier ${ }^{1,2,3,4}$, E. Cucchetti ${ }^{5}$, L. Tornatore ${ }^{6}$, V. Biffi ${ }^{7,8}$, E. Pointecouteau ${ }^{5}$, N. Clerc $^{5}$, P. Peille ${ }^{9}$, E. Rasia ${ }^{6,8}$, \\ D. Barret ${ }^{5}$, S. Borgani ${ }^{6,10,11,8}$, E. Bulbul ${ }^{12,13}$, T. Dauser ${ }^{14}$, K. Dolag ${ }^{15,16}$, S. Ettori ${ }^{17,18}$, M. Gaspari ${ }^{19,17, \star}$, F. Pajot ${ }^{5}$, \\ M. Roncarelli ${ }^{17,20}$, and J. Wilms ${ }^{14}$
}

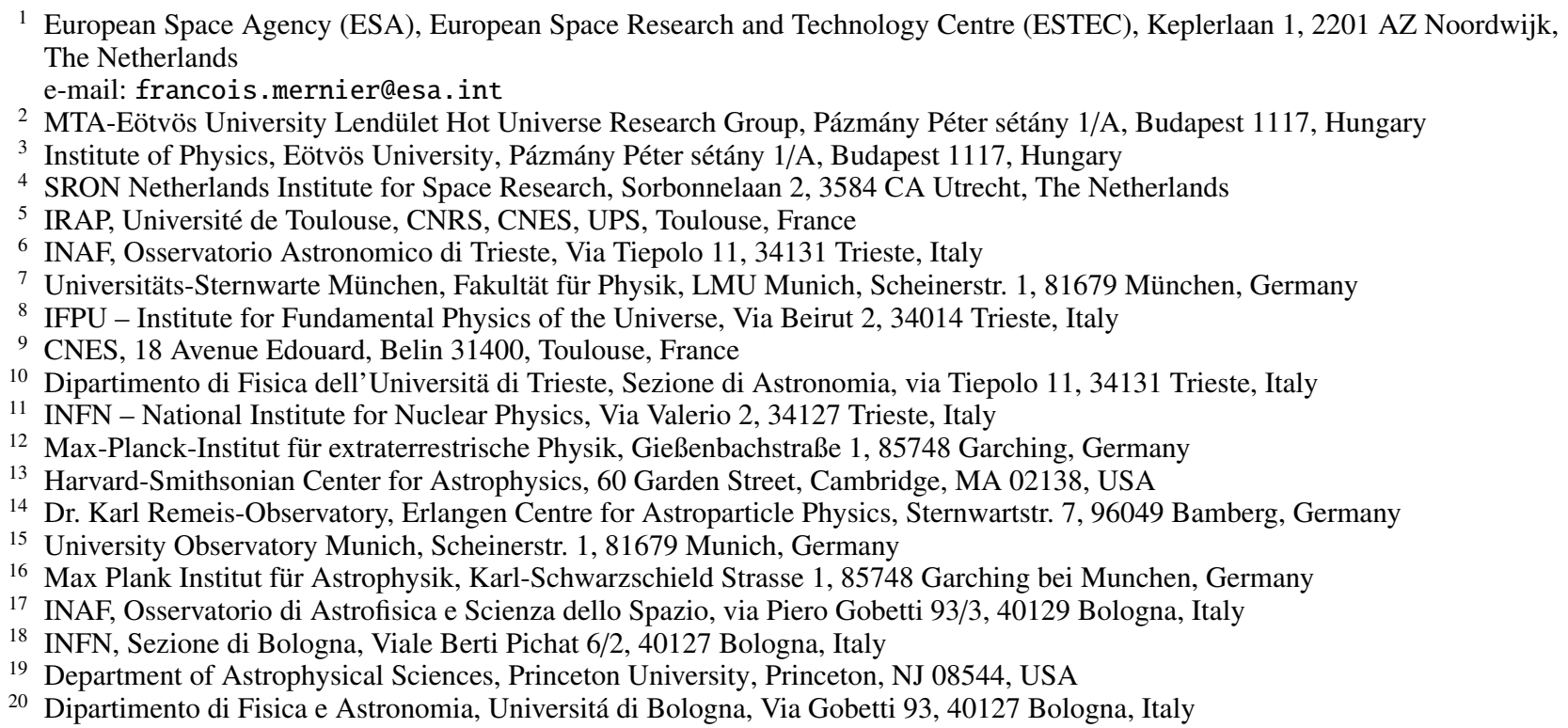

Received 11 June 2020 / Accepted 30 July 2020

\section{ABSTRACT}

\begin{abstract}
Chemical enrichment of the Universe at all scales is related to stellar winds and explosive supernovae phenomena. Metals produced by stars and later spread throughout the intracluster medium (ICM) at the megaparsec scale become a fossil record of the chemical enrichment of the Universe and of the dynamical and feedback mechanisms determining their circulation. As demonstrated by the results of the soft X-ray spectrometer onboard Hitomi, high-resolution X-ray spectroscopy is the path to differentiating among the models that consider different metal-production mechanisms, predict the outcoming yields, and are a function of the nature, mass, and/or initial metallicity of their stellar progenitor. Transformational results shall be achieved through improvements in the energy resolution and effective area of X-ray observatories, allowing them to detect rarer metals (e.g. Na, Al) and constrain yet-uncertain abundances (e.g. C, Ne, Ca, Ni). The X-ray Integral Field Unit (X-IFU) instrument onboard the next-generation European X-ray observatory Athena is expected to deliver such breakthroughs. Starting from $100 \mathrm{ks}$ of synthetic observations of 12 abundance ratios in the ICM of four simulated clusters, we demonstrate that the X-IFU will be capable of recovering the input chemical enrichment models at both low $(z=0.1)$ and high $(z=1)$ redshifts, while statistically excluding more than $99.5 \%$ of all the other tested combinations of models. By fixing the enrichment models which provide the best fit to the simulated data, we also show that the $\mathrm{X}$-IFU will constrain the slope of the stellar initial mass function within $\sim 12 \%$. These constraints will be key ingredients in our understanding of the chemical enrichment of the Universe and its evolution.
\end{abstract}

Key words. X-rays: galaxies: clusters - galaxies: clusters: intracluster medium - galaxies: abundances - supernovae: general galaxies: fundamental parameters - methods: numerical

\section{Introduction}

The processes that lead to chemical enrichment of the Universe remain one of the major open questions in astrophysics. Most of the light elements $(\mathrm{H}, \mathrm{He}, \mathrm{Li})$ were produced in the very first minutes of the Universe, during the primordial nucleosynthesis (Cyburt et al. 2016). Metals (i.e. elements heavier than Li)

\footnotetext{
^ Lyman Spitzer Jr. Fellow.
}

are instead more recent, as most of this enrichment is related to supernovae events (SNe) and to stellar winds (Burbidge et al. 1957). In fact, different types of stellar sources do not produce the same elements. Elements from $\mathrm{O}$ to $\mathrm{Si}$ are predominantly produced by fusion reactions in the outer shells of massive stars $\left(M \geq 10 M_{\odot}\right)$ during core-collapse supernovae $\left(\mathrm{SN}_{\mathrm{cc}}\right.$, see Nomoto et al. 2013 for a review), while heavier elements (i.e. $\mathrm{Si}$ to $\mathrm{Fe}$ ) are mostly related to thermonuclear explosions of white dwarfs (WDs) - former remnants of low-mass stars 
$\left(M \leq 8 M_{\odot}\right)$ - that is, type-Ia supernovae $\left(\mathrm{SN}_{\text {Ia }}\right.$, see Maoz et al 2014 for a review). Lighter elements (e.g. C, N, O) can also be related to radiative stellar winds when low-mass stars enter their asymptotic giant branch (AGB) phase (Iben \& Renzini 1983).

Though understood to some extent, the physics of these mechanisms is not fully constrained. For instance, two main scenarios compete to explain $\mathrm{SN}_{\mathrm{Ia}}$ events: the explosive C-burning onto a WD (triggering the $\mathrm{SN}_{\mathrm{Ia}}$ explosion) may be ignited either by (i) the accretion of matter from a companion star when the WD mass approaches the Chandrasekhar mass (singledegenerate model, Whelan \& Iben 1973), or (ii) the merger with another WD far below its Chandrasekhar mass (doubledegenerate model, Webbink 1984). The nature of the WD explosion itself also remains unclear (Hillebrandt et al. 2013) and endof-life models invoke either deflagration or delayed-detonation scenarios to explain observations (Iwamoto et al. 1999). Similarly, for $\mathrm{SN}_{\mathrm{cc}}$, metal production depends on the initial mass (hence lifetime) and metallicity of the star, $Z_{\text {init }}$, the estimation of which is challenging. Beyond the progenitor mass, chemical enrichment at all scales is strongly coupled to the initial mass function (IMF), i.e. the relative proportion of low- and highmass stars that are born within a given single stellar population. In fact, different IMFs result in different (relative) numbers of AGBs, $\mathrm{SN}_{\mathrm{cc}}$, and $\mathrm{SN}_{\mathrm{Ia}}$, affecting not only the chemical properties of galaxies, but also their entire evolution and feedback (for a review, see e.g. Bastian et al. 2010). However, whether the IMF is the same for all galaxies or constant with time is still an open question (e.g. De Masi et al. 2019), and therefore observational signatures of the IMF at galactic scales and beyond are valuable in this respect.

Measurements through X-ray spectroscopy of the intracluster medium (ICM) performed by missions such as $X M M$ Newton, Chandra, or Suzaku provide outstanding results in recovering the chemical composition of the ICM and in probing the enrichment of the largest scales of the Universe (for recent reviews, see Werner et al. 2008; Mernier et al. 2018a). In fact, the investigation of radial metallicity profiles in the outskirts of these systems (Werner et al. 2013; Urban et al. 2017) highlighted strong evidence of an early metal-production scenario $(z>2-3)$, which predates the formation of clusters (Fabjan et al. 2010; McCarthy et al. 2010; Biffi et al. 2017, 2018a, for a review on metallicity profiles in numerical simulations, see Biffi et al. $2018 \mathrm{~b}$ ) and is likely contemporary to the stelliferous epoch of the Universe (Madau \& Dickinson 2014). Although active galactic nucleus (AGN) feedback of the dominant galaxy can in principle induce significant central metallicity variations as a function of outflow and/or jet events (e.g. Gaspari et al. 2011), the remarkable similarity in the spatial distribution of $\mathrm{SN}_{\mathrm{Ia}}$ and $\mathrm{SN}_{\mathrm{cc}}$ products even within the central metallicity peaks suggests a similar "early-enrichment" scenario for clusters (and groups) cores (Simionescu et al. 2009; Million et al. 2011; Mernier et al. 2017). Nevertheless, the exact diffusion and transport mechanisms of metals from stars to the interstellar medium and beyond remains an open question in both observations (Kirkpatrick et al. 2011; De Grandi et al. 2016; Urdampilleta et al. 2019) and simulations (e.g. Schindler \& Diaferio 2008; Greif et al. 2009).

Additional information can be derived from the abundance ratios measured in the ICM. For example, the $\mathrm{Mn} / \mathrm{Fe}$ and $\mathrm{Ni} / \mathrm{Fe}$ ratios are both sensitive to the electron capture rates during $\mathrm{SN}_{\text {Ia }}$ explosions, and are therefore crucial indicators of their progenitor channels (Seitenzahl et al. 2013a; Mernier et al. 2016a; Hitomi Collaboration 2017), while ratios of lighter elements can in principle provide constraints on the IMF and the initial metallicity of the $\mathrm{SN}_{\mathrm{cc}}$ progenitors (e.g. de Plaa et al. 2007;
Mernier et al. 2016a). By pushing current observatories to their limit, recent studies derived constraints on the relative fraction of SN events that effectively contribute to the ICM enrichment. These measurements showed that $\mathrm{SN}_{\mathrm{Ia}}$ and $\mathrm{SN}_{\mathrm{cc}}$ contribute relatively equally to the overall chemical enrichment in the ICM (Werner et al. 2006; de Plaa et al. 2006, 2017; Bulbul et al. 2012; Mernier et al. 2016a). The comprehensive study of Simionescu et al. (2019) compiled the most accurate abundance measurements of the Perseus cluster (taken with the XMMNewton RGS and Hitomi SXS instruments) and compared them to state-of-the-art $\mathrm{SN}_{\mathrm{Ia}}$ and $\mathrm{SN}_{\mathrm{cc}}$ yield models. Their surprising conclusion is that no current set of models is able to reproduce all the observed abundance ratios at once. In particular, the measured $\mathrm{Si} / \mathrm{Ar}$ ratio tends to be systematically overpredicted by models, even when taking calibration and atomic uncertainties into account. Whereas further improvement of stellar nucleosynthesis models is expected, the non-negligible systematic errors associated to these observations and the lack of highly sensitive, spatially resolved high-resolution spectroscopy prevents us from steering any considerable change in the paradigm (see also de Grandi \& Molendi 2009). In fact, measurements from currently flying missions are performed with moderate collective area, either over the whole X-ray band (0.4-10 keV) with modest spectral resolution $(>100 \mathrm{eV})$, or with higher resolution dispersive spectroscopy but over the low $\mathrm{E}$ band $(0.3-2 \mathrm{keV})$ and without any spatial resolution, which considerably limits interpretations.

As revealed by Hitomi SXS (Takahashi et al. 2018), more accurate measurements of rare elements (e.g. $\mathrm{Ne}, \mathrm{Cr}$ or $\mathrm{Mn})$ will be key to constraining $\mathrm{SN}$ models. These steps forward are expected through the successor of the SXS, Resolve, on the X-Ray Imaging and Spectroscopy Mission (XRISM, XRISM Science Team 2020), but definitive answers will require instruments such as the X-ray Integral Field Unit (X-IFU, Barret et al. 2016, 2018) which will fly onboard Athena (Nandra et al. 2013). With more than 3000 micro-calorimeter pixels, the X-IFU will provide high-resolution spectroscopy on the $0.2-12 \mathrm{keV}$ energy band $(2.5 \mathrm{eV}$ FWHM energy resolution out to $7 \mathrm{keV}$ ) over an equivalent diameter of $5^{\prime}$ with $5^{\prime \prime}$ pixel size. This will (i) allow us to measure for the first time abundances of rare elements (Ettori et al. 2013a), (ii) provide unprecedented constraints on the spatial distribution of metals through the ICM (Cucchetti et al. 2018, hereafter, Paper I), and (iii) allow us to further explore the chemical evolution of the ICM down to $z \sim 2$ (Pointecouteau et al. 2013). Other astrophysical questions on the ICM, specifically its level and distribution of turbulence (e.g. Roncarelli et al. 2018; Clerc et al. 2019; Cucchetti et al. 2019), will also be explored by the X-IFU in unprecedented detail.

In this paper, we perform a first investigation of the capabilities of the X-IFU in constraining the sources of the ICM chemical enrichment and the associated stellar IMF in galaxy clusters. To do so, we use inputs from hydrodynamical cosmological simulations as already adopted in Paper I. The methods are described in Sect. 2. Using the large number of theoretical models at our disposal, we first demonstrate the capabilities of the X-IFU in recovering the underlying enrichment mechanisms assumed in the simulations (Sect. 3). By fixing the best-fit models to the data, a second study is performed to analyse the capabilities of the instrument in constraining important parameters linked to the IMF, such as its slope, shape, and the high-mass cut-off (Sect. 4). Results obtained in both cases are then discussed and future prospects are addressed (Sect. 5). Finally, we conclude our findings in Sect. 6. 
Throughout this paper, we assume that metals showing emission lines in the X-ray band are produced by three independent astrophysical sources: AGBs, $\mathrm{SN}_{\mathrm{cc}}$, and $\mathrm{SN}_{\mathrm{Ia}}$. The abundances and their associated ratios (i.e. normalised to the $\mathrm{Fe}$ abundance) are given in the solar units of Anders \& Grevesse (1989). Although other reference tables are available in the literature (e.g. the proto-solar, meteoritic abundances of Lodders et al. 2009), the choice of units here has no impact on our results, as long as they are used self-consistently from the simulations to the mock data analysis. We also assume a $\Lambda$-CDM cosmology with $\Omega_{\mathrm{M}}=0.24, \Omega_{\mathrm{b}}=0.04, H_{0}=72 \mathrm{~km} \mathrm{~s}^{-1} \mathrm{Mpc}^{-1}$, $\sigma_{8}=0.8$ and $n_{s}=0.96$ as used in the original hydrodynamical simulations (Rasia et al. 2015). Unless stated otherwise, the quoted errors consider a $68 \%$ confidence level.

\section{Methods and simulations}

\subsection{Simulation setup and mock data}

As current measurements are not adequate in terms of joint spatial and spectral resolution to build a representative toy model and perform a feasibility study of the afore-presented science case by X-IFU, this study relies on input numerical simulations, which are then converted into mock X-IFU datasets. The full method is described extensively in Paper I (to which we refer the reader for more details).

In summary, four Lagrangian regions are extracted from a parent large-scale cosmological simulation and re-simulated at higher resolution including the treatment of hydrodynamical processes with sub-grid physics models (using the treePM smoothed particle hydrodynamics code GADGET-3; see Rasia et al. 2015). Of these four systems, two are "cool-core" and two are "non-cool-core", and each class contains both a lowand a high-mass cluster. Each of these four clusters is traced back successively to $z=0.1$ and $z=1$. Metal enrichment by AGBs, $\mathrm{SN}_{\mathrm{cc}}$, and $\mathrm{SN}_{\mathrm{Ia}}$ is implemented following the approach of Tornatore et al. (2007), in which yields for $\mathrm{H}, \mathrm{He}, \mathrm{C}, \mathrm{N}, \mathrm{O}, \mathrm{Ne}$, $\mathrm{Na}, \mathrm{Mg}, \mathrm{Al}, \mathrm{Si}, \mathrm{S}, \mathrm{Ar}, \mathrm{Ca}, \mathrm{Fe}$, and $\mathrm{Ni}$ are injected and tracked during the clusters evolution (Biffi et al. 2018a; Truong et al. 2019). These elements are assumed to be produced by (i) AGBs, (ii) $\mathrm{SN}_{\mathrm{cc}}$, and (iii) $\mathrm{SN}_{\mathrm{Ia}}$ following the yields of, respectively, (i) Karakas (2010), (ii) Woosley \& Weaver (1995) with updates from Romano et al. (2010), and (iii) Thielemann et al. (2003).

These yield models are listed in boldface in Tables A.1A.3. They are used as input in our cosmological simulations to estimate the abundance of the different metal species produced during the evolution of the stellar component, assuming proper lifetimes (Padovani \& Matteucci 1993) and depending on its metallicity and mass distributions. For the latter, the simulations assume a Chabrier IMF of the stellar population (Chabrier 2003, see also Sect. 4). For this reason, most of the produced $\alpha$-elements are the result of a complex distribution of $\mathrm{AGB}$ and $\mathrm{SN}_{\mathrm{cc}}$ initial metallicities (Tornatore et al. 2007), from which we aim further to recover the dominant contribution only. X-ray photons ultimately emitted by these simulations are then projected and converted into event lists suitable for synthetic observations with the X-IFU for fixed exposure time of $100 \mathrm{ks}$. This is done using the end-to-end simulator SIXTE (Dauser et al. 2019) ${ }^{1}$ and assuming a vvapec model for the parameters of each emitting particle (for a similar approach, see also Roncarelli et al. 2018). As SIXTE is the official simulator for the X-IFU, the up-to-date response files of the instrument are used. For each of the nearby $(z=0.1)$ systems, we

\footnotetext{
1 https://www.sternwarte.uni-erlangen.de/research/ sixte/
}

simulate seven adjacent X-IFU pointings in order to fully cover their $R_{500}$ limits.

We analyse the mock data following Paper I. Specifically, the projected mock spectra are extracted within $R_{500}$ for each cluster, and are then fitted with XSPEC (Arnaud 1996) within $0.2-12 \mathrm{keV}$, using the same vvapec as in the input simulations for consistency (with the normalisation, temperature, redshift, and relevant abundances as free parameters). This approach thus neglects the impact of the uncertainties in the atomic models, which could be ultimately a limiting factor at the sensitivity to be reached by the X-IFU (for further discussion on systematic uncertainties and our approach to dealing with them, see Sects. 3.2 and 5.3). Other typical observational effects are taken into account. In particular, the implementation of the background relies on a modelling of the astrophysical foreground (consisting of the local hot bubble and the Milky Way hot gaseous content, modelled by an unabsorbed and absorbed thermal plasma, respectively), the cosmic X-ray background (unresolved AGNs, modelled by a power law), and the instrumental background (directly implemented within SIXTE). More details about this procedure are given in Paper I. The obtained best-fit abundance ratios are shown in Table 1 for each individual cluster as well as for the sample average (which are used throughout this paper). They can be directly compared to their corresponding input values from the simulations described above (listed on the same table). Results for other parameters, in particular the temperature, are further detailed in Paper I.

\subsection{Production yields and principle of the comparison}

The origin of metals in the ICM can be traced by $K$ distinct stellar sources of enrichment $k$ (in our case, $K=3$ and $k$ refers successively to AGBs, $\mathrm{SN}_{\mathrm{cc}}$, and $\mathrm{SN}_{\mathrm{Ia}}$ ). As such, the total number of atoms, $N_{X, \text { tot }}$, of a given chemical element $X$ produced over time can be expressed as (Gastaldello \& Molendi 2002):

$N_{X, \mathrm{tot}}=\sum_{k=1}^{K} n_{k} N_{X, k}$

where $n_{k}$ are multiplicative constants representing the total number of each source needed to obtain the observed enrichment (i.e. number of $\mathrm{SNe}$ or $\mathrm{AGBs}$ ), and $N_{X, k}$ represents the number of atoms produced by each source. This number can easily be related to the mass $M_{X, k}$ of a produced element as usually provided by yield models from the literature:

$N_{X, k}=\frac{M_{X, k}}{\mu_{X}}$

where $\mu_{X}$ is the atomic weight of the element $X$ (in the same units as $M_{X, k}$ ). Usually, however, AGBs and $\mathrm{SN}_{\mathrm{cc}}$ yield models are provided for a specific progenitor mass $m$ and need to be integrated over a single stellar population with a given IMF. Such an integrated mass $M_{X, k}^{\text {int }}$, assuming a power-law IMF, can be written as:

$$
M_{X, k}^{\mathrm{int}}=\frac{\int_{M_{\mathrm{low}}}^{M_{\mathrm{low}}} M_{X, k}(m) m^{\alpha} \mathrm{d} m}{\int_{M_{\mathrm{low}}}^{M_{\mathrm{cut}}} m^{\alpha} \mathrm{d} m},
$$

where $\alpha$ is the slope of the IMF, $M_{\text {low }}$ is the lowest stellar mass assumed for each source of enrichment (depending on the yield models, though typically $\sim 1 M_{\odot}$ and $\sim 10 M_{\odot}$ for AGBs 
Table 1. Mean abundance ratios with respect to iron, obtained from the simulation input values ("i" in the second column) and the analysis of the mock X-IFU data ("o" in the second column) of the $z \sim 0.1$ simulated clusters of Paper I (100 ks of mock exposure for each system, labelled as cool-core $[\mathrm{CC}]$ or non-cool-core $[\mathrm{NCC}]$ ).

\begin{tabular}{lcccccc}
\hline \hline Ratio & $\begin{array}{c}\text { Input (i) / } \\
\text { observed (o) }\end{array}$ & $\begin{array}{c}\text { Cluster 1 } \\
(\mathrm{CC})\end{array}$ & $\begin{array}{c}\text { Cluster 2 } \\
\text { (NCC) }\end{array}$ & $\begin{array}{c}\text { Cluster 3 } \\
(\mathrm{CC})\end{array}$ & $\begin{array}{c}\text { Cluster 4 } \\
(\mathrm{NCC})\end{array}$ & Sample average \\
\hline $\mathrm{C} / \mathrm{Fe}$ & $\mathrm{i}$ & 0.50 & 0.51 & 0.49 & 0.50 & 0.50 \\
& $\mathrm{o}$ & $0.48 \pm 0.34$ & $0.35 \pm 0.24$ & $0.43 \pm 0.28$ & $0.48 \pm 0.31$ & $0.39 \pm 0.15$ \\
$\mathrm{~N} / \mathrm{Fe}$ & $\mathrm{i}$ & 0.39 & 0.40 & 0.40 & 0.39 & 0.39 \\
& $\mathrm{o}$ & $0.32 \pm 0.12$ & $0.40 \pm 0.15$ & $0.52 \pm 0.30$ & $0.57 \pm 0.20$ & $0.41 \pm 0.08$ \\
$\mathrm{O} / \mathrm{Fe}$ & $\mathrm{i}$ & 0.71 & 0.66 & 0.62 & 0.73 & 0.68 \\
& $\mathrm{o}$ & $0.73 \pm 0.04$ & $0.68 \pm 0.04$ & $0.67 \pm 0.06$ & $0.69 \pm 0.06$ & $0.69 \pm 0.02$ \\
$\mathrm{Ne} / \mathrm{Fe}$ & $\mathrm{i}$ & 0.11 & 0.11 & 0.11 & 0.12 & 0.12 \\
& $\mathrm{o}$ & $0.10 \pm 0.03$ & $0.12 \pm 0.04$ & $0.14 \pm 0.06$ & $0.20 \pm 0.06$ & $0.12 \pm 0.02$ \\
$\mathrm{Na} / \mathrm{Fe}$ & $\mathrm{i}$ & 0.12 & 0.12 & 0.13 & 0.13 & 0.13 \\
& $\mathrm{o}$ & $0.17 \pm 0.12$ & $0.34 \pm 0.24$ & $0.20 \pm 0.13$ & $0.21 \pm 0.17$ & $0.21 \pm 0.08$ \\
$\mathrm{Mg} / \mathrm{Fe}$ & $\mathrm{i}$ & 0.61 & 0.61 & 0.62 & 0.61 & 0.61 \\
& $\mathrm{o}$ & $0.63 \pm 0.05$ & $0.58 \pm 0.06$ & $0.59 \pm 0.08$ & $0.53 \pm 0.08$ & $0.59 \pm 0.03$ \\
$\mathrm{Al} / \mathrm{Fe}$ & $\mathrm{i}$ & 0.14 & 0.11 & 0.11 & 0.16 & 0.13 \\
& $\mathrm{o}$ & $0.16 \pm 0.08$ & $0.18 \pm 0.10$ & $0.21 \pm 0.18$ & $0.20 \pm 0.13$ & $0.18 \pm 0.06$ \\
$\mathrm{Si} / \mathrm{Fe}$ & $\mathrm{i}$ & 1.20 & 1.21 & 1.22 & 1.20 & 1.21 \\
& $\mathrm{o}$ & $1.24 \pm 0.06$ & $1.18 \pm 0.05$ & $1.27 \pm 0.08$ & $1.27 \pm 0.09$ & $1.23 \pm 0.03$ \\
$\mathrm{~S} / \mathrm{Fe}$ & $\mathrm{i}$ & 1.30 & 1.28 & 1.25 & 1.22 & 1.26 \\
& $\mathrm{o}$ & $1.33 \pm 0.08$ & $1.30 \pm 0.08$ & $1.26 \pm 0.12$ & $1.18 \pm 0.12$ & $1.28 \pm 0.05$ \\
$\mathrm{Ar} / \mathrm{Fe}$ & $\mathrm{i}$ & 0.23 & 0.23 & 0.23 & 0.23 & 0.23 \\
& $\mathrm{o}$ & $0.19 \pm 0.09$ & $0.22 \pm 0.11$ & $0.20 \pm 0.15$ & $0.30 \pm 0.17$ & $0.22 \pm 0.06$ \\
$\mathrm{Ca} / \mathrm{Fe}$ & $\mathrm{i}$ & 0.93 & 0.94 & 0.94 & 0.93 & 0.93 \\
& $\mathrm{o}$ & $0.94 \pm 0.20$ & $0.71 \pm 0.16$ & $0.71 \pm 0.24$ & $0.99 \pm 0.25$ & $0.82 \pm 0.10$ \\
$\mathrm{Ni} / \mathrm{Fe}$ & $\mathrm{i}$ & 2.17 & 2.11 & 2.23 & 2.17 & 2.17 \\
& $\mathrm{o}$ & $2.12 \pm 0.20$ & $1.91 \pm 0.21$ & $2.11 \pm 0.29$ & $2.12 \pm 0.32$ & $2.05 \pm 0.12$ \\
\hline
\end{tabular}

Notes. All values are within $R_{500}$.

and $\mathrm{SN}_{\mathrm{cc}}$, respectively ${ }^{2}$ ), and $M_{\text {cut }}$ is the upper mass cut-off for the integration (yet uncertain, though typically assumed to be $\left.\sim 40 M_{\odot}\right)$. In the input numerical simulations, the actual IMF is represented by a Chabrier-like piece-wise power-law function with $M_{\text {cut }}=40 M_{\odot}$ and the following slopes: $\alpha=-1.2$ for $M \in[0.1,0.3] M_{\odot}, \alpha=-1.8$ for $M \in[0.3,1.3] M_{\odot}$, and $\alpha=-2.3$ for $M \in[1.3,40] M_{\odot}$. The Salpeter-like IMF (often used in previous literature as well as in this paper) follows the slope $\alpha=-2.35$ and is very similar to the Chabrier-like IMF beyond $\sim 1 M_{\odot}$.

The respective "absolute" (i.e. relative to $\mathrm{H}$ ) abundance $\mathcal{A}_{X, k}$ of a given element $X$ is defined as:

$\mathcal{A}_{X, k} \equiv \frac{N_{X, k} / N_{\mathrm{H}, k}}{\left(N_{X} / N_{\mathrm{H}}\right)_{\mathrm{ref}}}=\frac{N_{X, k}}{N_{\mathrm{H}, k} \mathcal{A}_{X, \mathrm{ref}}}$,

where $\mathcal{A}_{X, \text { ref }} \equiv\left(N_{X} / N_{\mathrm{H}}\right)_{\text {ref }}$ are the selected solar (or proto-solar) reference abundances (in our case, Anders \& Grevesse 1989).

Given the above equations, the associated $X / \mathrm{Fe}$ abundance ratio (i.e. relative to $\mathrm{Fe}$ ) can be expressed as:

$\mathcal{Y}_{X, k} \equiv \frac{\mathcal{A}_{X, k}}{\mathcal{A}_{\mathrm{Fe}, k}}=\frac{M_{X, k}}{M_{\mathrm{Fe}, k}} \frac{\mu_{\mathrm{Fe}}}{\mu_{X}} \frac{\mathcal{A}_{\mathrm{Fe}, \mathrm{ref}}}{\mathcal{A}_{X, \mathrm{ref}}}$.

Considering now all the sources of enrichment, and similar to Eq. (1), the overall abundance ratio $\mathcal{Y}_{X \text {,tot }}$ can be written

2 Below $\sim 1 M_{\odot}$, stellar mass losses are negligible compared to the AGB wind losses above that limit. Below $\sim 10 M_{\odot}$, the core of a star is not massive enough to collapse and trigger a $\mathrm{SN}_{\mathrm{cc}}$ explosion (e.g. Nomoto et al. 2013).
$\boldsymbol{y}_{X, \text { tot }}=\sum_{k=1}^{K} a_{k} \boldsymbol{y}_{X, k}$,

where $a_{k}$ is another multiplicative constant (linked to $n_{k}$ with appropriate normalisation). By definition $\boldsymbol{y}_{\mathrm{Fe} \text {,tot }}=1$.

The previous equations are valid in the case of an overall study of the abundances, which accounts for the total number of atoms produced in stars, galaxies, and the ICM (Matteucci \& Chiappini 2005). However, X-ray observations provide a direct measurement of the ICM content in heavy elements. In addition, while the previous approach is rather insensitive to the spatial variation of metals within the ICM itself (due to, e.g. turbulence or diffusion - as clusters within $R_{500}$ are considered as closed-boxes), it does not consider the circulation timescale of metals from stars into the ICM (see also de Grandi \& Molendi 2009), nor the possibly different locations of $\mathrm{AGB}, \mathrm{SN}_{\mathrm{cc}}$, and $\mathrm{SN}_{\mathrm{Ia}}$ due to the different lifetimes of their progenitors. It does not account either for any potential difference in the metal distribution (relative and spatial differences) between the ICM phase and the stellar phase. A usual caveat, assumed here, is to consider that the fractions $a_{k}$ derived from ICM studies are representative of the number of events enriching the ICM-only system, rather than the total galaxy cluster system (i.e. stellar and ICM phases). In this case, the previous formula can be applied treating $\mathcal{Y}_{X, k}$ as "effective" yields, which describe the fractions of the stellar sources enriching the hot gas (equivalent if and only if the metal distribution is the same in galaxies and the ICM, Humphrey \& Buote 2006).

Using X-ray measurements of abundance ratios, $\tilde{y}_{X}$, the consistency of a theoretical prediction of abundance ratios can be 
tested at the ICM level by fitting a linear combination of the integrated yields that minimises

$\chi^{2}=\sum_{X} \frac{\left(\tilde{\boldsymbol{y}}_{X}-\boldsymbol{y}_{X, \mathrm{tot}}\right)^{2}}{\sigma_{\text {stat }, X}^{2}}$,

where the sum is performed over the total number of available elements $X$, and $\sigma_{\text {stat }}$ is the statistical error of the measurements $\tilde{\mathcal{Y}}_{X}$ (our strategy to deal with potential systematic errors is explained further in Sect. 3.1). The recovered values of $a_{k} / \sum_{k=1}^{K} a_{k}$ represent the corresponding fractions of each source $k$ at play in the ICM enrichment.

A generic way of quantifying the accuracy of a model in describing observations is to compute the reduced chi-squared of the fit, $\chi_{\text {red }}^{2}$. If large values are obtained, the predicted yields are not likely to represent the observations of the chemical enrichment (at least at the ICM level) in a realistic way. These results can also be further refined by applying additional observational constraints on the ratio of $\mathrm{SN}_{\mathrm{Ia}}$-to-SNe (e.g. Mernier et al. 2016a). Current observational studies are limited in this comparison by the large (statistical and/or systematic) in the measured abundance ratios, and the limited number of elements observed (e.g. Mernier et al. 2015, 2016b). As shown in Table 1, X-IFU observations will provide a wealth of new constraints to discard or verify certain models. By comparing our mock data (Sect. 2.1) to a significant number of available models, in the following sections we derive which set of models matches the ratios in the most statistically accurate way, assessing whether it is ultimately possible to recover the models originally used in the input simulation (described in detail in Tornatore et al. 2007; Biffi et al. 2017).

\section{Constraining chemical enrichment models with the X-IFU}

Besides the three distinct and independent sources of enrichment considered here - $\mathrm{AGB}, \mathrm{SN}_{\mathrm{cc}}$, or $\mathrm{SN}_{\mathrm{Ia}}$, we also assume that the bulk of the enrichment is completed at $z=0.1$ and does not differ from local clusters, which is a fair hypothesis supported by numerical and observational results (e.g. Ettori et al. 2015; McDonald et al. 2016; Biffi et al. 2017, 2018b; Urban et al. 2017; Mantz et al. 2017; Liu et al. 2020). Section 3.4 further extends the comparison to the case of $z=1$ clusters.

In the following sections, nucleosynthesis yields computed for various models from the literature are fitted to the averaged $X / \mathrm{Fe}$ abundance ratios measured by the synthetic $\mathrm{X}$-IFU observations. This is done by using the abunfit package in python ${ }^{3}$, which solves the minimisation problem in Eq. (7) for $a_{\mathrm{SN}_{\mathrm{Ia}}}, a_{\mathrm{SN}_{\mathrm{cc}}}, a_{\mathrm{AGB}}$ (based on the approach of Ettori et al. 2002; Gastaldello \& Molendi 2002; Werner et al. 2006; de Plaa et al. 2007; Mernier et al. 2016a, and Simionescu et al. 2019). The fit is then performed under the constraint:

$a_{\mathrm{AGB}} \mathcal{Y}_{\mathrm{Fe}, \mathrm{AGB}}+a_{\mathrm{SN}} \mathcal{y}_{\mathrm{Fe}, \mathrm{SN}_{\mathrm{cc}}}+a_{\mathrm{SN}_{\mathrm{Ia}}} \mathcal{Y}_{\mathrm{Fe}, \mathrm{SN}_{\mathrm{Ia}}}=1$.

The full list of models (along with their relevant specifics) used for this comparison, and covering a significant fraction of the recent literature, is detailed in Appendix A. Most AGB and $\mathrm{SN}_{\mathrm{cc}}$ models are tested individually over the available range of initial metallicities $Z_{\text {init }}$. Among the tested $\mathrm{SN}_{\mathrm{cc}}$ models, we also include a set of hypernova and pair-instability SN predicted yields in order to explore the reproducibility of the abundance

\footnotetext{
https://github.com/mernier/abunfit
}

pattern through an enrichment from very massive, metal-poor stars. To ease the comparison, throughout this section AGB and $\mathrm{SN}_{\mathrm{cc}}$ yields are first integrated using the same Salpeterlike power-law IMF with a slope $\alpha=-2.35$ (Salpeter 1955). Although, formally, not exactly the same as the Chabrier-like IMF used in the input simulations, the Salpeter-like parametrisation is reasonable as a first approximation, is the simplest one to use, and is usually considered in previous similar studies. Other choices of the IMF (including Chabrier-like, which marginally improves our fit) are explored in Sect. 4. A summary of our fits and methods is shown in Table 2.

\subsection{Recovering the input simulated enrichment models}

As a first safety check, we aim to recover our X-IFU mock observed ratios with the combinations of the yield models that were considered as input in the simulated clusters. This corresponds to all the "K10+Ro10+Th03" combinations as listed in Appendix A (4 AGB models $\times 5 \mathrm{SN}_{\mathrm{cc}}$ models $\times 1 \mathrm{SN}_{\mathrm{Ia}}$ model $=$ 20 combinations).

Figure 1 shows the best-fit abundance pattern under these assumptions, obtained with $Z_{\text {init }}=0.01 Z_{\odot}$ for $\mathrm{SN}_{\mathrm{cc}}$ yields and $Z_{\text {init }}=1 Z_{\odot}$ for AGB yields ${ }^{4}$. With only two element ratios not perfectly recovered $(\mathrm{Mg} / \mathrm{Fe}$ and $\mathrm{Ni} / \mathrm{Fe})$, the agreement between the mock ratios and best-fit models is good, leading to $\chi_{\text {red }}^{2}=$ 1.49. This is particularly reassuring as under our assumptions it demonstrates the remarkable ability of the X-IFU to reproduce the true chemical composition of the ICM. We note that the exquisite precision achieved on most ratios naturally inflates $\chi_{\text {red }}^{2}$ for the elements whose yields are not perfectly recovered. In fact, when ignoring the $\mathrm{Mg} / \mathrm{Fe}$ and $\mathrm{Ni} / \mathrm{Fe}$ ratios, the fit further improves with $\chi_{\text {red }}^{2}=0.55$. The precise reasons of the $>1 \sigma$ discrepancies on these two specific ratios may be various (e.g. numerical issues, projection effects ${ }^{5}$, contribution from less dominant $Z_{\text {init }}$ as initially implemented) and are left for investigation in future work.

\subsection{Constraints on various enrichment models}

Going one step further, though still assuming that each source of enrichment can be described by one single model, we aim to test whether the observed abundance pattern can be (mis-) interpreted with other combinations of $\mathrm{AGB}+\mathrm{SN}_{\mathrm{cc}}+\mathrm{SN}_{\mathrm{Ia}}$ yield models available from the literature. This is particularly important in order to demonstrate the ability of the X-IFU to constrain relevant astrophysical parameters on the stellar population itself (e.g. average initial metallicity of $\mathrm{SN}_{\mathrm{cc}}$ progenitors, favoured explosion channel of $\mathrm{SN}_{\mathrm{Ia}}$ ).

For each combination of models (i.e. a total of 4 AGB models $\times 24 \mathrm{SN}_{\mathrm{cc}}$ models $\times 182 \mathrm{SN}_{\mathrm{Ia}}$ models $=17472 \mathrm{com}-$ binations), we computed the reduced chi-squared of the fit with 8 degrees of freedom (corresponding to 12 elements -3 models - 1). The corresponding $\chi_{\text {red }}^{2}$ of each combination, along with

\footnotetext{
4 As stellar evolution within our input simulations provided various initial metallicities for AGB and $\mathrm{SN}_{\mathrm{cc}}$ progenitors, the best-fit $Z_{\text {init }}$ values reported here translate their average contribution to the enrichment. 5 As seen from Figs. 5 and 8 of Paper I, the simulation-to-data discrepancies of the $\mathrm{Mg} / \mathrm{Fe}$ ratio are mainly present in Cluster 3 (cool-core), while the other systems do not seem to be affected. While this may suggest that projection effects play a role in such simulations-to-data discrepancies (especially if Cluster 3 is affected by large-scale motions or substructures), the absence of such discrepancies for other ratios originating from the same source of enrichment (e.g. $\mathrm{O} / \mathrm{Fe}$ ) prevents us from excluding the other possibilities mentioned in the text.
} 
Table 2. Summary of the fits performed on our mock observations throughout this study.

\begin{tabular}{lccccc}
\hline \hline Section & $z$ & Combination & Free parameters & d.o.f. & Total number of comb. \\
\hline 3.1 & 0.1 & $\left(\mathrm{AGB}+\mathrm{SN}_{\mathrm{cc}}+\mathrm{SN}_{\mathrm{Ia}}\right)_{\text {input }}$ & $2\left(\mathrm{SN}_{\mathrm{Ia}} / \mathrm{SNe}, \mathrm{AGB} / \mathrm{SNe}\right)$ & 8 & $4 \times 5 \times 1=20$ \\
3.2 & 0.1 & $\mathrm{AGB}+\mathrm{SN}_{\mathrm{cc}}+\mathrm{SN}_{\mathrm{Ia}}$ & $2\left(\mathrm{SN}_{\mathrm{Ia}} / \mathrm{SNe}, \mathrm{AGB} / \mathrm{SNe}\right)$ & 8 & $4 \times 24 \times 182=17472$ \\
3.3 & 0.1 & $\mathrm{AGB}+\mathrm{SN}_{\mathrm{cc}}+\mathrm{SN}_{\mathrm{Ia}, 1}+\mathrm{SN}_{\mathrm{Ia}, 2}$ & $3\left(\mathrm{SN}_{\mathrm{Ia}, 1} / \mathrm{SN}_{\mathrm{Ia}, 1+2}, \mathrm{SN} \mathrm{Ia}, 1+2 / \mathrm{SNe}, \mathrm{AGB} / \mathrm{SNe}\right)$ & 7 & $4 \times 24 \times 78 \times 96=718848$ \\
3.4 & 1 & $\left(\mathrm{AGB}+\mathrm{SN}_{\mathrm{cc}}+\mathrm{SN}_{\mathrm{Ia}}\right)_{\text {input }}$ & $2\left(\mathrm{SN}_{\mathrm{Ia}} / \mathrm{SNe}, \mathrm{AGB} / \mathrm{SNe}\right)$ & 6 & $4 \times 5 \times 1=20$ \\
\hline
\end{tabular}

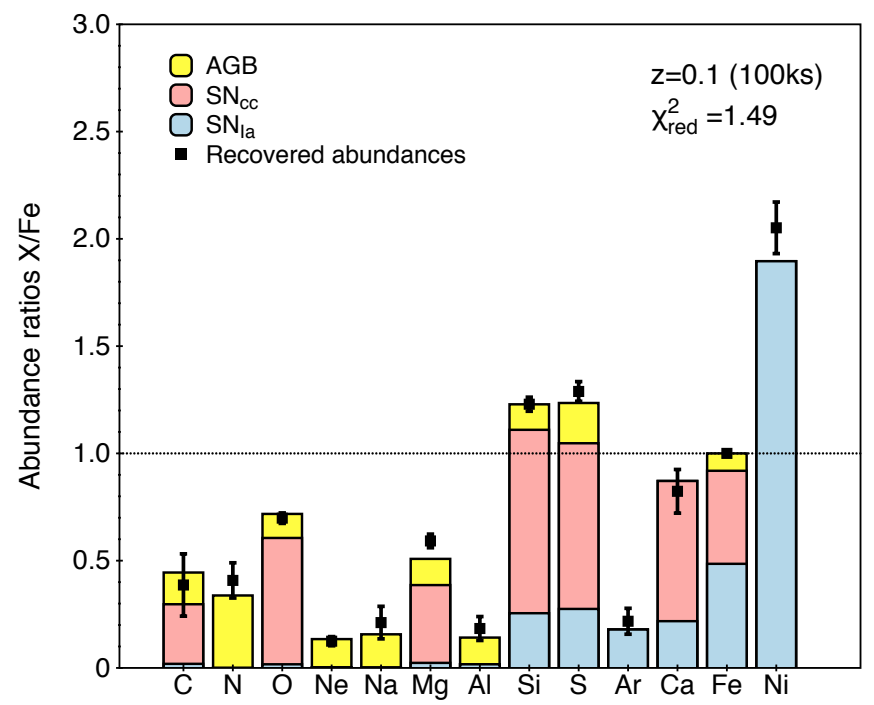

Fig. 1. Average abundance ratio $X /$ Fe within $R_{500}$ over the cluster sample for $z=0.1$ recovered using $100 \mathrm{ks}$ observations. These values are fitted using the enrichment yields derived from the references provided in Paper I. The corresponding fitted contributions of $\mathrm{SN}_{\mathrm{Ia}}$ (blue), $\mathrm{SN}_{\mathrm{cc}}$ (magenta), and AGB stars (yellow) are shown as histograms.

the associated best-fit $\mathrm{SN}_{\mathrm{Ia}}$ fraction - i.e. $\mathrm{SN}_{\mathrm{Ia}} / \mathrm{SNe}$ or, more formally, $a_{\mathrm{SN}_{\mathrm{Ia}}} /\left(a_{\mathrm{SN}_{\mathrm{cc}}}+a_{\mathrm{SN}_{\mathrm{Ia}}}\right)-$ is shown in Fig. 2 (left). All these combinations are also counted in histograms and their distribution is shown in Fig. 2 (right). We define a combination as "statistically acceptable" if it verifies $\chi_{\text {red }}^{2} \lesssim 5$. Such a conservative value is chosen arbitrarily to account for systematic error in the models or in the IMF and to avoid ruling out potentially significant models. The distribution indicates that, in the vast majority of cases (17383), the fit does not provide a good description of the mock measurements $\left(\chi_{\text {red }}^{2}>5\right)$. In fact we can distinguish two different features. A first peak $\left(\chi_{\text {red }}^{2} \sim 30\right)$ corresponds to a fit in which one out of the fitted models (either $\mathrm{SN}_{\mathrm{c}}$ or $\mathrm{SN}_{\mathrm{Ia}}$ ) is different from the input family of models used to perform the cosmological simulations. In that case, two of the models are close to reproducing correct metal production yields but the third one is not. Likewise, the second peak $\left(\chi_{\text {red }}^{2} \sim 80\right)$ corresponds to the case where both the $\mathrm{SN}_{\mathrm{cc}}$ and the $\mathrm{SN}_{\mathrm{Ia}}$ models are different from the input family of models. In that case, none of them is consistent with the data set, thus providing a poorly accurate description of our mock abundance pattern.

From the list of models presented in Appendix A, only 89 $\mathrm{AGB}+\mathrm{SN}_{\mathrm{cc}}+\mathrm{SN}_{\mathrm{Ia}}$ combinations provide statistically acceptable results $\left(\chi_{\text {red }}^{2} \lesssim 5\right)$. This represents only $0.5 \%$ of the total number of combinations. The X-IFU is therefore able to reject more than $\geq 99 \%$ of the theoretical combinations of models tested here. A further refinement can be performed by analysing the results of the fit, in particular the relative contribution of each mechanism. From previous observations, we know that the observed $\mathrm{SN}_{\text {Ia }}$ fraction is, very conservatively, comprised between 0.1 and 0.6 (see Tables 5 and 6 in de Grandi \& Molendi 2009). As such, we can constrain the number of statistically accurate fits even further by requesting that $a_{\mathrm{SN}} /\left(a_{\mathrm{SN}_{\mathrm{Ia}}}+a_{\mathrm{SN}_{\mathrm{cc}}}\right)$ remains within these limits (Fig. 2, left). Following this second selection, 53 combinations satisfy $\chi_{\text {red }}^{2}<5$. In Table 3 , we show the ten best-fit combinations satisfying this criterion.

While the combination of models used in the numerical simulations and recovered in Sect. 3.1 is remarkably situated among the top of all these best-fit combinations (black arrow in Fig. 2, left), we note that two other combinations (using respectively the 100-3-c3 and 500-5-c3 $\mathrm{SN}_{\mathrm{Ia}}$ models from Leung \& Nomoto 2018) provide a slightly better $\chi_{\text {red }}^{2}$. We discuss the reasons for this mis-interpretation in Sect. 5.1 and further demonstrate that in more realistic conditions the X-IFU will be easily able to refine the discriminations between these combinations and strongly favour the one corresponding to the genuine sources of enrichment.

Among these statistically acceptable results, we also note that all the combinations predict correctly the input family of $\mathrm{SN}_{\mathrm{cc}}$ models. In fact, the yellow circles of Fig. 2 (left) below the dashed red line are all combinations in which only the $\mathrm{SN}_{\mathrm{Ia}}$ model was not consistent with the input simulations. This means that our fits are more inclined to recover an accurate $\mathrm{SN}_{\mathrm{cc}}$ (family of) model(s) rather than an accurate $\mathrm{SN}_{\text {Ia }}$ model. However, the ability of the X-IFU to measure more ratios than presented here (Sect. 5.1) will eventually result in a much tighter filtering on the $\mathrm{SN}_{\mathrm{Ia}}$ models.

Despite the conservative treatment of the systematic uncertainties considered above, one may wonder how our results would be altered if the X-IFU abundance measurements of one of the key elements turns out to be unreliable. To check this scenario, we re-fit our 17472 combinations ignoring successively one given $X / \mathrm{Fe}$ ratio. The main effect is a slight decrease of $\chi_{\text {red }}^{2}$ without significantly altering the $\mathrm{SN}_{\mathrm{Ia}}$ fraction, resulting in a horizontal left shift of the pattern seen in Fig. 2 (left). Ignoring Ni/Fe has the most noticeable impact, with a total of 443 combinations becoming statistically acceptable (i.e. $\sim 2.5 \%$ of the total number of combinations). This is not surprising as, among all the ratios tested here, $\mathrm{Ni} / \mathrm{Fe}$ is by far the most efficient at separating different $\mathrm{SN}_{\mathrm{Ia}}$ models (see our discussion in Sect. 5.1). Ignoring other key ratios, such as $\mathrm{Si} / \mathrm{Fe}$ or $\mathrm{O} / \mathrm{Fe}$ (respectively 138 and 126 "statistically acceptable" combinations), has a much less pronounced impact on our results. Providing that the X-IFU will be able to measure the abundances of other key heavy elements (e.g. Cr, Mn; Sect. 5.1), our conclusions therefore hold even if not all ratios were considered as reliable.

\subsection{Lifting model degeneracies: the case of two $S N_{\text {Ia }}$ models}

In theory, multiple sources of the same type may co-exist and enrich the ICM in comparable amounts. This might be notably the case for $\mathrm{SN}_{\text {Ia }}$, whose end of life can be significantly different depending on the single-degenerate versus 
Table 3. Top ten best-fit combinations of $\mathrm{AGB}+\mathrm{SN}_{\mathrm{cc}}+\mathrm{SN}_{\mathrm{Ia}}$ models with our X-IFU mock observed abundance ratios at $z=0.1$, with $0.1<$ $\mathrm{SN}_{\mathrm{Ia}} / \mathrm{SNe}<0.6$ (see text).

\begin{tabular}{lclccc}
\hline \hline$\chi_{\text {red }}^{2}$ & AGB model & $\mathrm{SN}_{\text {cc }}$ model & $\mathrm{SN}_{\text {Ia }}$ model & $\mathrm{SN}_{\mathrm{Ia}}$ frac. & AGB frac. \\
\hline 1.314 & K10_0.02 & Ro10_2E-3 & $100-3-c 3$ & 0.430 & 0.340 \\
1.366 & K10_0.02 & Ro10_2E-3 & $500-5-c 3$ & 0.420 & 0.330 \\
$1.490^{(\dagger)}$ & K10_0.02 & Ro10_2E-4 & Th03 & 0.452 & 0.319 \\
1.591 & K10_0.02 & Ro10_2E-3 & $100-5-c 3$ & 0.431 & 0.328 \\
1.722 & K10_0.02 & Ro10_2E-3 & $300-5-c 3$ & 0.431 & 0.325 \\
1.982 & K10_0.02 & Ro10_2E-3 & $500-3-c 3$ & 0.376 & 0.328 \\
2.182 & K10_0.02 & Ro10_2E-4 & $100-3-c 3$ & 0.463 & 0.337 \\
2.505 & K10_0.008 & Ro10_2E-3 & $500-5-c 3$ & 0.393 & 0.388 \\
2.583 & K10_0.02 & Ro10_2E-3 & $300-3-c 3$ & 0.372 & 0.325 \\
2.594 & K10_0.008 & Ro10_2E-4 & Th03 & 0.429 & 0.377 \\
\hline
\end{tabular}

Notes. The models mentioned here are all further detailed in Appendix A. The models used as input in the cosmological simulations are given in bold. ${ }^{(\dagger)}$ This model combination is considered fixed in Sect. 4.
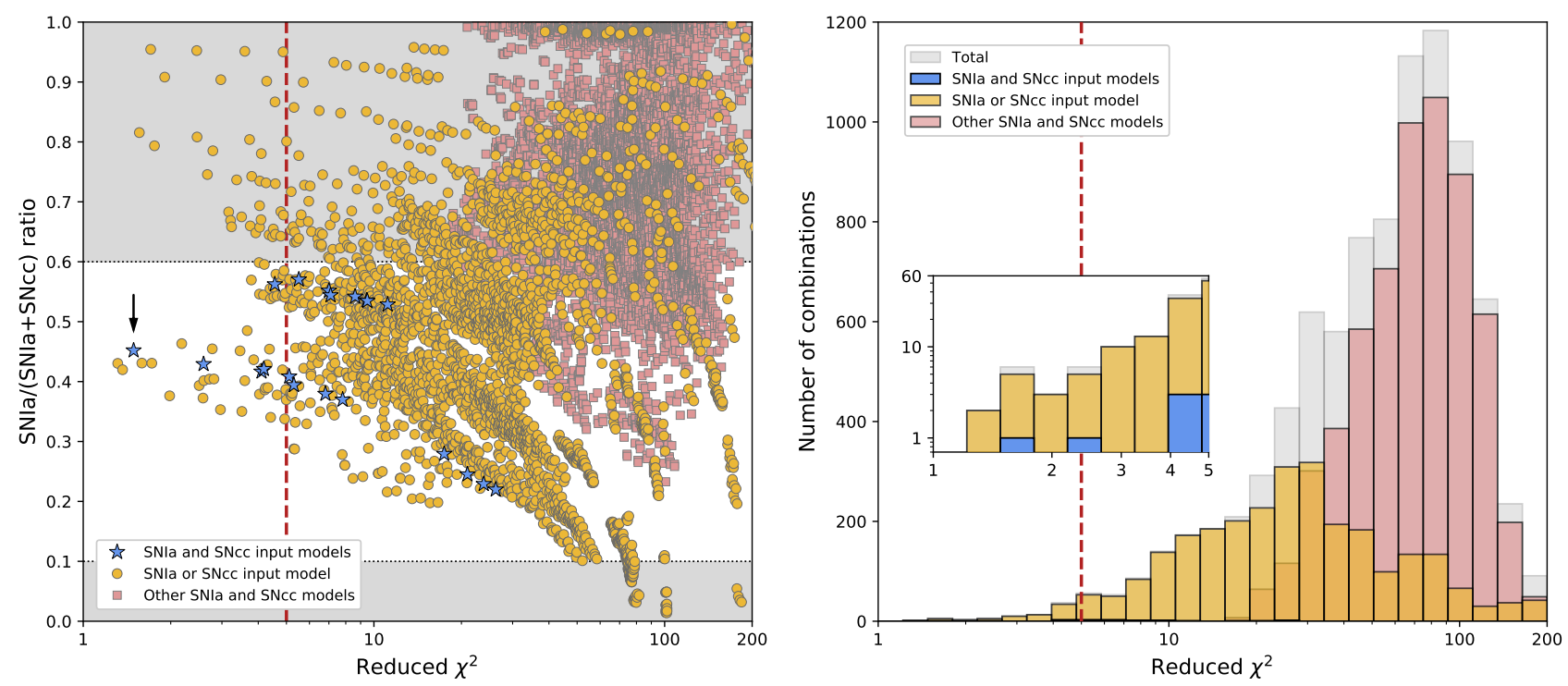

Fig. 2. Left: best-fit $\chi_{\text {red }}^{2}$ versus the $\mathrm{SN}_{\mathrm{Ia}}$ fraction for all the possible combinations of $\left(\mathrm{AGB}+\mathrm{SN}_{\mathrm{cc}}+\mathrm{SN}_{\mathrm{Ia}}\right)$ yield models, fitted on the averaged $\mathrm{X}$-IFU mock abundance ratios (see text). Combinations using (i) both $\mathrm{SN}_{\mathrm{cc}}$ and $\mathrm{SN}_{\mathrm{Ia}}$ models from the input cosmological simulations (blue stars), (ii) one of these models (for either $\mathrm{SN}_{\mathrm{cc}}$ or $\mathrm{SN}_{\mathrm{Ia}}$; yellow circles), and (iii) neither of these models (pink squares) are shown separately. The black arrow shows the "input" combination detailed in Fig. 1 and discussed in Sect. 3.1. The white area delimitates realistic observational constraints previously obtained on the $\mathrm{SN}_{\mathrm{Ia}}$ fraction contributing to the enrichment (see text). The dashed red line shows the "statistically acceptable" models $\left(\chi_{\text {red }}^{2} \leq 5\right.$, see text). The list of best-fit models is provided in Table 3. Right: $\chi_{\text {red }}^{2}$ distribution of the same combinations. The same colour code as the left panel is applied.

double-degenerate scenario of their progenitors (and/or their deflagration vs. delayed-detonation thermonuclear explosions). Although this possibility is worth exploring, the use of multiple models to represent one class of physical events introduces one additional degree of freedom, which can be degenerate if the number and accuracy of the observed ratios is limited. In fact, this is one of the major limitations of current observatories when testing enrichment scenarios (Mernier et al. 2016a). To verify whether the accuracy expected from future X-IFU measurements is able to lift this degeneracy, we included an additional $\mathrm{SN}_{\text {Ia }}$ model to the fit to represent a more complex stellar reality. The same method as in Eq. (8) (Sect. 3) is applied, considering two scalars, $a_{\mathrm{SN}}$ Ia, 1 and $a_{\mathrm{SN}_{\mathrm{Ia}}, 2}$, in the fit. Specifically, one $\mathrm{SN}_{\mathrm{Ia}}$ model is chosen to be near-Chandrasekhar (near- $M_{\mathrm{Ch}}$, corresponding predominantly to the single-degenerate progenitor channel) while the other is chosen to be sub-Chandrasekhar (sub- $M_{\mathrm{Ch}}$, corresponding predominantly to the double-degenerate progen- itor channel; see also Table A.3). Assuming the same diversity of $\mathrm{AGB}$ and $\mathrm{SN}_{\mathrm{cc}}$ models as in the previous section, this corresponds to a total of $4 \mathrm{AGB}$ models $\times 24 \mathrm{SN}_{\mathrm{cc}}$ models $\times 78$ $\mathrm{SN}_{\text {Ia }, 1}$ models $\times 96 \mathrm{SN}_{\text {Ia, } 2}$ models $=718848$ combinations of models. Although our input simulations include only one (near$M_{\mathrm{Ch}}$ ) $\mathrm{SN}_{\mathrm{Ia}}$ contribution (Sect. 2.1), this exercise is fully relevant as it shows whether or not the X-IFU measurement accuracies are good enough to avoid misinterpreting one dominant $\mathrm{SN}_{\mathrm{Ia}}$ model with a combination of two $\mathrm{SN}_{\mathrm{Ia}}$ models that would (incorrectly) contribute in comparable amounts to the enrichment.

We find that 472 of these $\mathrm{AGB}+\mathrm{SN}_{\mathrm{cc}}+\mathrm{SN}_{\mathrm{Ia}, 1}+\mathrm{SN}_{\mathrm{Ia}, 2}$ combinations offer a $\chi_{\text {red }}^{2}$ value below 1.5 (13 of which contain one input $\mathrm{SN}_{\text {Ia }}$ model), and are thus better at reproducing our X-IFU mock abundance pattern than the input $\mathrm{AGB}+\mathrm{SN}_{\mathrm{cc}}+\mathrm{SN}_{\mathrm{Ia}}$ set of models (Sect. 3.1, Fig. 1). Although this seems to be a large number, this represents only $\sim 0.07 \%$ of the total number of our combinations including two SNIa models (near- $M_{\mathrm{Ch}}+\mathrm{sub}-M_{\mathrm{Ch}}$ ). 


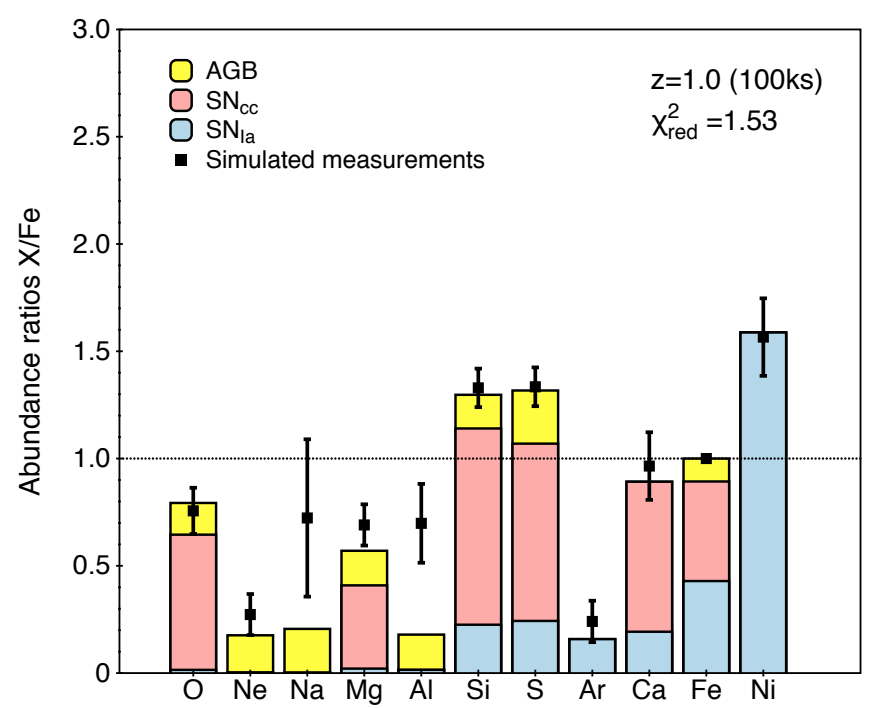

Fig. 3. Same as Fig. 1, over the cluster sample at $z=1$ (instead of $z=0.1$ ). Carbon and nitrogen are not shown as the lines are outside the energy bandpass of the X-IFU instrument.

The more complex scenario where two SNIa models co-exist can be further constrained imposing a limit on the relative contributions from the two competing mechanisms. For instance, we can request the $\mathrm{SN}_{\mathrm{Ia}, 1} / \mathrm{SN}_{\mathrm{Ia}, 2}$ ratio (namely, $a_{\mathrm{SN}_{\mathrm{Ia}}, 1} / a_{\mathrm{SN}_{\mathrm{Ia}}, 2}$ ) not to exceed one order of magnitude, that is, that it lies between 0.1 and 10. These limits are arbitrarily chosen to satisfy the condition that both mechanisms remain quantitatively comparable (otherwise a single model should suffice at first order). When applying this criterion, together with the same criterion as in Sect. 3.2 (i.e. $0.1<\mathrm{SN}_{\mathrm{Ia} 1+2} / \mathrm{SNe}<0.6$ ), the number of "realistic" combinations below $\chi_{\text {red }}^{2}<1.5$ drops to 190 (i.e. $\sim 0.03 \%$ of the total number of combinations).

Admittedly, the present case includes more degeneracies than our previous, less complex attempts (i.e. simple $\mathrm{AGB}+\mathrm{SN}_{\mathrm{cc}}+\mathrm{SN}_{\mathrm{Ia}}$ combinations). These numbers, and their interpretation as to the ability of the X-IFU to disentangle an ICM enrichment from one or two (or more) $\mathrm{SN}_{\mathrm{Ia}}$ models, are further discussed in Sect. 5.1.

\subsection{Best fits and extension to higher redshift}

The results shown above offer very promising perspectives for the X-IFU. Through simple linear fits and physical considerations, we are able to accurately recover the underlying enrichment model implemented in the hydrodynamical simulations. As shown in Fig. 1 and Sect. 3.1, the best fit for the data set is obtained, at $z=0.1$, for $Z_{\text {init }}=0.01 Z_{\odot}$ for $\mathrm{SN}_{\mathrm{cc}}$ yields and $Z_{\text {init }}=1 Z_{\odot}$ for AGB yields, with values of the reduced chisquared of 1.49 for local clusters, nicely recovering the input yield models from the simulations.

The previous approach is also extended at $z=1$ with excellent results (see Fig. 3). We find that the X-IFU is still capable of accurately recovering abundance ratios within $R_{500}$ and the underlying input combination of models (same as Sect. 3.1) with good accuracy $\left(\chi_{\text {red }}^{2}=1.53\right)$. In addition, similarly to what has been reported in the input simulations (Biffi et al. 2018a), our mock X-IFU observations consistently show no significant changes in the measured ratios between our two tested redshifts.

We note that the aim of this exercise is limited to recovering the yields used in our input simulations. A full constraint among all the other considered models (as in Sect. 3.2 and Sect. 3.3) is indeed less relevant at higher redshift if, as in our simulations, one assumes no substantial change in the physics of $\mathrm{AGB}, \mathrm{SN}_{\mathrm{cc}}$, and $\mathrm{SN}_{\mathrm{Ia}}$ within the last 7-8 Gyr of ICM enrichment. Moreover, In the case of high-redshift systems, low-mass elements such as $\mathrm{C}$ and $\mathrm{N}$ can no longer be detected (as their emission lines fall outside the X-ray energy window) and measuring the abundance of rarer elements (e.g. $\mathrm{Ne}, \mathrm{Na}$, and $\mathrm{Al}$ ) with sufficient statistical accuracy will require exposures deeper than $100 \mathrm{ks}$. At those high redshifts and for $100 \mathrm{ks}$ exposures (or less), the derived AGB fraction is characterised by large uncertainties because the weight of those key low-mass elements becomes minimal in the fit. More accurate results call for better-adapted exposure strategies and larger sample studies in order to optimise the results for distant objects and to investigate the chemical enrichment of the $\mathrm{AGB}$ (in addition to $\mathrm{SNe}$ ) across cosmic time.

\section{Recovering the IMF with the X-IFU}

\subsection{Effect of the IMF on yields}

Nucleosynthesis yields for $\mathrm{AGB}$ and $\mathrm{SN}_{\mathrm{cc}}$ are usually given for a specific progenitor mass. As a consequence, they need to be integrated in a consistent (and realistic) way before fitting the result to our measurements (together with $\mathrm{SN}_{\text {Ia }}$ models). In the approach described above, in order to be consistent with the large majority of previous observational strategies (de Plaa et al. 2007; Mernier et al. 2016a; Simionescu et al. 2019), full integration of these yields is performed using a Salpeter-like power law. Though representative at first order, more advanced models can be used (e.g. Chabrier-like, as in our input simulations; Chabrier 2003) to provide a more accurate description of the low-mass parts of the IMF in the integration. Similarly, the mass cut-off beyond which a massive star directly collapses into a black hole (i.e. without ejecting freshly produced elements, and therefore not influencing $\mathrm{AGB}$ and $\mathrm{SN}_{\mathrm{cc}}$ products) can be varied in the integration. Since all our models are integrated using the same IMF and mass cut-off (for consistency), no such related effects are expected in the above results. However, for a given $\mathrm{AGB}+\mathrm{SN}_{\mathrm{cc}}+\mathrm{SN}_{\mathrm{Ia}}$ combination, one can vary $\alpha$ and $M_{\text {cut }}$ and study their effects on the fits. This is particularly relevant to verify whether (and to which extent) the X-IFU will be able to provide constraints on the shape, the slope, and the mass cut-off of the IMF.

\subsection{Recovering the IMF parameters for a fixed set of models}

The original IMF proposed by Salpeter assumes a value of $\alpha=-2.35$ (Salpeter 1955). As our simulations initially assumed a Chabrier-like IMF, we aim to explore the effects of such changes on our best-fit combination of input yields (i.e. K10_0.02+Ro10_2E-4+Th03, see Sect. 3.1) at $z=0.1$. To do so, we re-integrate the total $\mathrm{AGB}$ and $\mathrm{SN}_{\mathrm{cc}}$ yields over various arbitrary values of the IMF (Eq. (3)), with $\alpha \in[-1.5,-3.0]$, and using cut-off values of $M_{\text {cut }} \in[25,50] M_{\odot}$. We then successively re-fit these modified models on our mock measurements. The results - namely the variation of $\chi_{\text {red }}^{2}$ on the $\left\{\alpha, M_{\text {cut }}\right\}$ parameter plane - are provided in Fig. 4. For the given degrees of freedom, we notice that only a limited region of this plane provides results that are statistically consistent (i.e. within the $68 \%$ and $95 \%$ confidence intervals, corresponding respectively to $\Delta \chi^{2}$ values of 9.30 and 15.51 for 8 degrees of freedom) with our initial assumptions. 


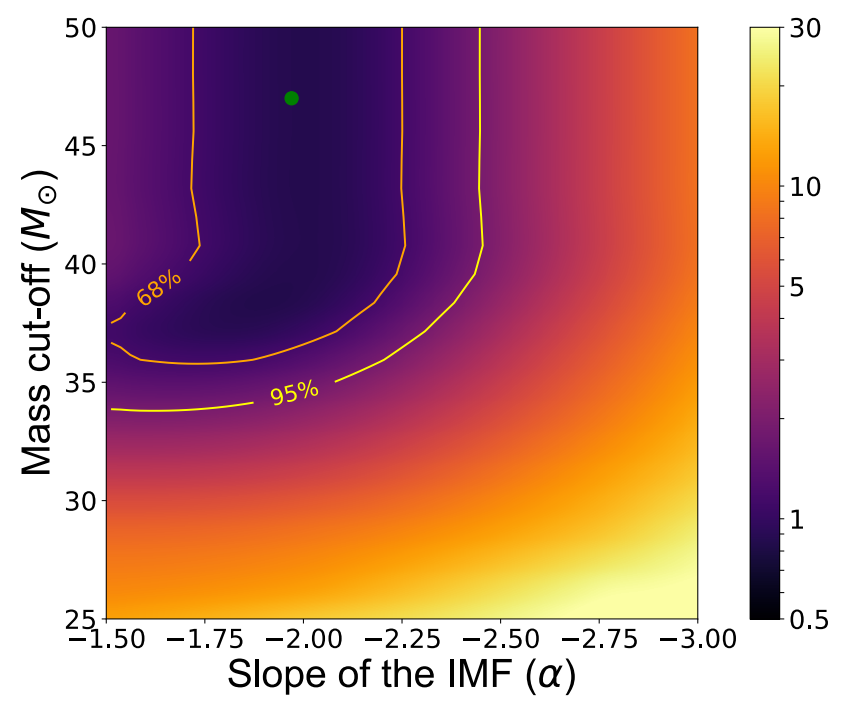

Fig. 4. Value of $\chi_{\text {red }}^{2}$ of the fit of the data provided in Table 1, with the best model from Table 3, for various Salpeter-like IMFs, as a function of the upper mass cut-off (in $M_{\odot}$ ) and slope of the power-law $(\alpha)$. The contours delimitate the $68 \%$ and $95 \%$ confidence levels for the given degrees of freedom (in orange and yellow, respectively). The best-fit value is indicated by the green $\operatorname{dot}\left(\alpha=-1.97, M_{\text {cut }}=47 M_{\odot}, \chi_{\text {red }}^{2}=\right.$ $0.87)$.

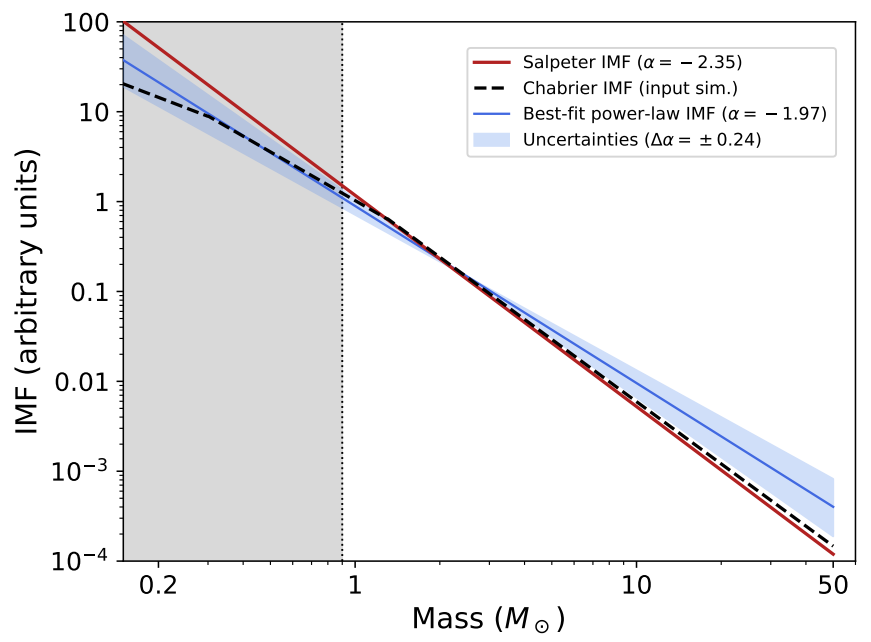

Fig. 5. Comparison of assumed slopes of the IMF throughout this study (normalised for their integral within [0.9-50] $M_{\odot}$ to be unity). The input Chabrier IMF (piece-wise function) as used in our input simulations is well reproduced by a power-law IMF of slope $\alpha=-1.97 \pm 0.24$ (see text). Stars with $<0.9 M_{\odot}$ are not expected to directly enrich their surroundings, and therefore IMFs are not considered below that mass limit.

Clearly, the slope of the IMF has a significant effect on the consistency of the fit. Depending on its value, the reduced chisquare rises from $\sim 1$ to more than 10 . The confidence interval contours show little dependence on the mass cut-off, such that the best-fit value is given by $\alpha=-1.97 \pm 0.24( \pm 0.47$ at the $95 \%$ level). Around these values, the mass cut-off of the IMF at low progenitor masses has a large effect on the yields. This is expected, as a low $M_{\text {cut }}$ implies that an appreciable fraction of massive stars is not considered in the integration, thereby causing a bias toward low values some low-mass elements. When $M_{\text {cut }} \geq 40 M_{\odot}$, the effect on the integration becomes negligible. This is related to the treatment of high-mass stars in the cosmo- logical simulations (Rasia et al. 2015), which are considered to collapse directly into black-holes beyond $40 M_{\odot}$, hence creating no chemical elements.

This best-fit value of the IMF slope $(\alpha \simeq-2)$ can also be explained (Fig. 5). When fitting the Chabrier-like piecewise function (Sect. 2.2) with a single slope power-law, the slope coefficient is indeed close to $\alpha \sim-2$. Although yields from AGBs (and by extension $\mathrm{SN}_{\mathrm{cc}}$ ) contribute only for progenitors above $0.9 M_{\odot}$, the same study as for the Salpeter-like IMF can in principle be performed also on Chabrier-like IMFs. When manually re-integrating the baseline yields (Sect. 3.1) with such a Chabrier-like IMF (as initially assumed in the original hydrodynamical simulations; Tornatore et al. 2007), we find slight improvements with respect to the best fits obtained so far (Sect. 3.1; Table 3). In fact, $\chi_{\text {red }}^{2}$ improves from 1.49 to 1.32 at $z=0.1$ (with the $\mathrm{S} / \mathrm{Fe}$ and $\mathrm{Ni} / \mathrm{Fe}$ ratios becoming $<1 \sigma$ consistent with the yield predictions, as well as a slight improvement on the $\mathrm{Mg} / \mathrm{Fe}$ ratio), and from 1.53 to 1.41 at $z=1$ (with improvements essentially on the $\mathrm{Na} / \mathrm{Fe}$ and $\mathrm{Al} / \mathrm{Fe}$ ratios).

Using very simple considerations on the IMF, we showed that the X-IFU will be able to provide useful constraints on the (average) IMF of the stellar population(s) responsible for the enrichment. To some extent, it will be capable of distinguishing the mass cut-off of this function and to provide refinements on the value of the power slope (especially in the high-mass regime of $\mathrm{SN}_{\mathrm{cc}}$ progenitors, where presumably the IMF is close to a single-slope power law). The observation of multiple clusters with the X-IFU and the accuracy of the recovered abundance ratios will provide an interesting tool for future IMF studies. Further discussion on this point and the ability of the X-IFU to favour different functional shapes of the IMF is provided in Sect. 5.2.

\section{Discussion}

\subsection{Ability of the X-IFU to constrain nucleosynthesis models}

In Sects. 3.1 and 3.2, we show that the combination of $\mathrm{AGB}+\mathrm{SN}_{\mathrm{cc}}+\mathrm{SN}_{\mathrm{Ia}}$ models representing the enrichment processes as injected in our cosmological simulations (blue stars in Fig. 2 left) are remarkably well recovered through the abundance ratios measured by the X-IFU. However, it also appeared that two other combinations (using $\mathrm{SN}_{\text {Ia }}$ models that were not initially considered in our simulations) provide a better fit to our mock abundance pattern.

At first glance, this may appear as a source of concern regarding the ability of the X-IFU to correctly isolate the dominant physical and environmental mechanisms at play for stellar sources responsible for the enrichment. Nevertheless, one should keep in mind that in the present exercise we limit our study to the elements individually tracked within the numerical simulations, while the capabilities of X-IFU (e.g. Ettori et al. 2013a) extend to the abundance measurement of other Fe-peak elements as well, such as Ti, V, Cr, and Mn. Although these elements were not tracked in the original simulations (Tornatore et al. 2007), they play a crucial role in the different $\mathrm{SN}_{\mathrm{Ia}}$ explosion and progenitor scenarios. We illustrate this effect in Fig. 6, where the predicted $X / \mathrm{Fe}$ ratios of the five most favoured $\mathrm{SN}_{\mathrm{Ia}}$ models from our $\mathrm{AGB}+\mathrm{SN}_{\mathrm{cc}}+\mathrm{SN}_{\mathrm{Ia}}$ fits (Table 3 ) are compared. It clearly appears that the $\mathrm{Ti} / \mathrm{Fe}, \mathrm{V} / \mathrm{Fe}, \mathrm{Cr} / \mathrm{Fe}$, and $\mathrm{Mn} / \mathrm{Fe}$ ratios offer a powerful way to favour and/or rule out specific models. On the other hand, it is quite remarkable to note that, in this work, the (already impressive) ability of the X-IFU to efficiently favour our input model (namely, Th03) among 181 other ones from the 


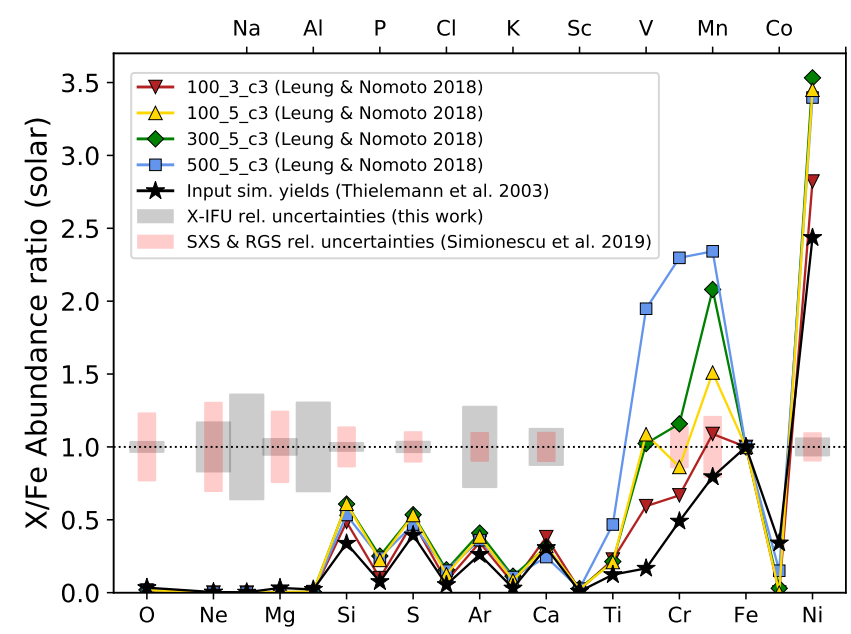

Fig. 6. Comparison of various $X / \mathrm{Fe}$ abundance ratios predicted by the $\mathrm{SN}_{\text {Ia }}$ yield models used in our top five " $\mathrm{SN}_{\text {Ia }}$ model" best fits (Table 3). Upper and lower horizontal axes mark respectively the odd- and even- $Z$ elements. For comparison, we also show the relative (i.e. normalised to 1) observational uncertainties on the $X / \mathrm{Fe}$ ratios derived (i) in this work using the X-IFU (12 ratios, as tracked by our input simulations, $\mathrm{C}$ and $\mathrm{N}$ are not shown here), and (ii) using $\sim 300 \mathrm{ks}$ of Hitomi/SXS (in synergy with $\sim 200 \mathrm{ks}$ of XMM-Newton/RGS) exposure (Simionescu et al. 2019).

literature was based solely on the $\mathrm{Si} / \mathrm{Fe}, \mathrm{S} / \mathrm{Fe}, \mathrm{Ar} / \mathrm{Fe}, \mathrm{Ca} / \mathrm{Fe}$, and $\mathrm{Ni} / \mathrm{Fe}$ ratios, which individually exhibit limited model-to-model differences.

These additional constraints expected from $\mathrm{Ti}, \mathrm{V}, \mathrm{Cr}$, and Mn will depend on the typical uncertainties that the X-IFU will measure for these abundances. While a complete re-run of cosmological simulations including these elements (along with a thorough mock spectral re-analysis) is out of the scope of this paper (see below), we highlight the remarkable constraints on $\mathrm{Cr} / \mathrm{Fe}$ and $\mathrm{Mn} / \mathrm{Fe}$ that were already achieved by the Hitomi SXS observations of the Perseus cluster (Hitomi Collaboration 2017; Simionescu et al. 2019, red areas in Fig. 6). Given the factor 10-12 improvement in weak-line sensitivity offered by the XIFU in that energy band (Barret et al. 2018) and the results presented in this work, it becomes clear that the X-IFU will ultimately be capable of isolating the most realistic physical and environmental constraints of SN progenitors responsible for the ICM enrichment.

The same reasoning could in principle be applied to the question of disentangling one from two $\mathrm{SN}_{\mathrm{Ia}}$ models (Sect. 3.3). Although the X-IFU is fully capable of favouring our input (single-SN $\mathrm{II}_{\mathrm{Ia}}$ ) enrichment model over more than $99.97 \%$ of all the combinations including two $\mathrm{SN}_{\mathrm{Ia}}$ (i.e. near- $M_{\mathrm{Ch}}+\mathrm{sub}-M_{\mathrm{Ch}}$ ) models, an ideal goal would consist of isolating the former while disfavouring all the two- $\mathrm{SN}_{\mathrm{Ia}}$ combinations. The inclusion of $\mathrm{Cr} / \mathrm{Fe}$ and $\mathrm{Mn} / \mathrm{Fe}$ ratios (and likely $\mathrm{Ti} / \mathrm{Fe}$ and $\mathrm{V} / \mathrm{Fe}$ ) will certainly help to approach this limit. As mentioned earlier, this is particularly relevant given the high sensitivity of $\mathrm{Mn}$ to disentangle between near- $M_{\mathrm{Ch}}$ and sub- $M_{\mathrm{Ch}}$ models (e.g. Seitenzahl et al. 2013a; Mernier et al. 2016a; Hitomi Collaboration 2017).

Admittedly, a complete demonstration of the X-IFU to favour one or two co-existing $\mathrm{SN}_{\mathrm{Ia}}$ models would require not only the inclusion of these additional elements in the input simulations, but also the testing of the inverse exercise as proposed here - i.e. inject two $\mathrm{SN}_{\text {Ia }}$ progenitor channels in the simulations and test whether combinations with one $\mathrm{SN}_{\text {Ia }}$ model all fail to reproduce the resulting abundance pattern. Due to limited com- puting capacity, such re-runs are beyond the scope of this study and deeper investigation is therefore left to future work.

Another point of importance has been mentioned in Simionescu et al. (2019), where the authors show that no combination of the current models can simultaneously reproduce all the measured abundance ratios in the Perseus cluster. This suggests that the current nucleosynthesis models need to be further improved to include more realistic physical processes that may have an impact on their ejected yields (see also de Grandi \& Molendi 2009). Such improvements will be crucial for the era of exquisite ICM abundance accuracies that will be unveiled by XRISM and Athena. They will also help to reduce the number of models that can be considered as physically realistic, further improving the constraints presented here.

\subsection{Constraints on the IMF}

The investigation on the IMF performed here (Sect. 4.2) demonstrates the breadth of possibilities that will be accessible through the X-IFU. In fact, most of the possible caveats come essentially from our choice of the enrichment models rather than the capabilities of the instrument. Specifically, we notice that with slight changes in the IMF parameters, $\chi_{\text {red }}^{2}$ can easily go above the previous threshold of five for our set of input models. The question of whether other sets of models could provide more accurate fits when integrated with slightly different IMFs thus naturally arises. However, integrating other models using modified IMFs (using the abunfit package) confirms the results seen in Sect. 4, that is, a best-fit slope typically around $\alpha \sim-2$ and $M_{\text {cut }} \sim 40 M_{\odot}$. This can be explained by the very simple shape of the IMF considered here, but could provide significantly different results with more complex functions.

In fact, the IMF considered in the fits is simplistic, but ensures that we have only two parameters (slope and mass cut-off) to ease the interpretation. Other shapes can be considered, especially those with lower solar mass roll-offs (notably Chabrier-like, Fig. 5), which assume a (realistic) finite number of stars at low masses. However, in this case, interpreting the contribution of each part of the IMF to the final integrated yields becomes more challenging. Furthermore, as most of these IMFs (see a few examples in Chabrier 2003) are approximated at high solar masses by a power law (similar to that of Salpeter 1955), no substantial change in the results is expected (mostly secondorder changes are observed when using a Chabrier-like IMF).

As shown in Sect. 4.2, the typical uncertainties on the ICM abundance ratios obtained with the $100 \mathrm{ks}$ of X-IFU exposure on four nearby clusters will therefore allow us to derive constraints on the slope of the IMF of the order of $\sim 12 \%$ (i.e. $\Delta \alpha=0.24)$. For comparison, while various measurements of the slope of the high-mass end of the IMF in the Galactic field, star-forming regions, associations, and star clusters show individual reported errors from $\sim 50 \%$ down to a few percent, they scatter with deviations up to $\sim 60-70 \%$ of the Salpeter value (e.g. Fig. 2 in Bastian et al. 2010). It becomes therefore evident that the Athena mission will play a key role in substantially improving our understanding of the IMF, its global properties, and the question of its universality. Given that elliptical galaxies show hot atmospheres even in compact, isolated halos (e.g. Reiprich \& Böhringer 2002; Kim et al. 2019; Lakhchaura et al. 2019a; Gaspari et al. 2019), and under the condition that plasma emission codes will further provide the necessary accuracy, similar IMF studies can be expanded to the low-mass regime with similar statistics (e.g. lower luminosity is compensated by higher line emissivity of $\alpha$-elements due to cooler plasma temperature). 
Because successive generations of stars become continuously enriched with metals (which may in turn affect their masses and lifetimes), it is also possible that the IMF evolves with cosmic time, with more weight toward massive stars at higher redshifts (van Dokkum 2008; Wang \& Dai 2011). For instance, van Dokkum (2008) reports a change of $\Delta \alpha \simeq 1.6$ between a sample of $0.02<z<0.83$ cluster galaxies and the canonical value around $1 M_{\odot}$. Although at a different mass range, this is larger than the typical uncertainty reported in Sect. 4.2. The question of how such a possible IMF evolution would affect the results presented here is not trivial, as it depends on (i) exactly how the IMF evolves with time and (ii) at which exact redshift range the metals seen today in the ICM were produced. While it is clear that good knowledge of the former will allow the X-IFU to bring constraints on the latter (or inversely), such constraints are difficult to quantify with the present simulations. Future work, with a time-changing IMF included in the input simulations, will certainly help to address this question.

As another consequence of stellar metallicities potentially evolving during the main epoch of enrichment, the simple assumption of using one $Z_{\text {init }}$ in our models may limit the interpretation of the above results. While in such a case one can reasonably expect that $Z_{\text {init }}$ represents the bulk of initial metallicities of $\mathrm{SN}_{\mathrm{cc}}$ progenitors, the precise impact on the derived IMF is less clear. As shown in Mernier et al. (2016a), the change of initial metallicity rather impacts the $\mathrm{O} / \mathrm{Ne}$ ratio (see their Fig. 2 right) while the effect of the IMF is rather reflected on the $\mathrm{Ne} / \mathrm{Mg}$ and $\mathrm{Ne} / \mathrm{Si}$ ratios (see their Fig. 8). Although at first order we speculate that these effects are therefore limited, dedicated studies will be necessary to further quantify them.

\subsection{Possible improvements of the current approach}

From the above results, it is clear that the exquisite accuracy of the X-IFU in deriving ICM abundance ratios will allow us to favour and/or rule out various nucleosynthesis scenarios and conditions to explain the enrichment in galaxy clusters. This will considerably improve the constraints offered by the current missions (e.g. de Plaa et al. 2007; Mernier et al. 2016a, 2018b) as well as with the available Hitomi observations (Simionescu et al. 2019). As this work is the first attempt to quantify such constraints with the X-IFU, a few more points of discussion should be addressed, specifically in the context of improving our future observing strategies.

First, and as outlined above, the obtained numbers (which are used to draw our conclusions) depend on the choice of the input yields in the hydrodynamical simulations used to generate synthetic X-IFU observations (described in Paper I and references therein). Different results might be expected if for instance the yields assumed in the hydrodynamical simulations produce a truly unique chemical signature while on the contrary a large fraction of all the other model combinations happen to produce very similar abundance patterns. Although this is in practice unlikely to be the case here (see the rather large spread in the $\chi_{\text {red }}^{2}$ of Fig. 2), a proper validation would require tests with other input models, which we defer to future studies. We also note that no uncertainties are associated with these yields, simply because none are directly available in the current literature (although de Grandi \& Molendi 2009 reported some yield uncertainties on the order of tens of percent).

In this study, abundance ratios were derived within $R_{500}$ over four clusters. Although no strong observational evidence has been reported so far, the internal dynamical structure of clusters (e.g. turbulence, diffusion, AGN feedback) could to some extent affect the abundance ratios with radial and/or azimuthal inhomogeneities. This would naturally induce biases as a function of the spatial scale over which measurements are performed. However, this effect can be safely neglected in our case. Indeed, Figs. 3, 4, and 5 of Paper I clearly show that despite mild spatial metallicity inhomogeneities for a given system (and mild clusterto-cluster differences in their metallicity profiles), the abundance ratios remain spatially uniform within uncertainties. It should be noted that, even though this picture is actually consistent with the absence of radial variation in abundance ratios reported on several observed systems (e.g. Ezer et al. 2017; Mernier et al. 2017) and in simulations (Biffi et al. 2017), future X-IFU observations will be able to simultaneously measure abundance ratios integrated over large regions with very high accuracy (as shown in this paper) and investigate abundance ratios on much smaller (1D or 2D) scales. These local variations and anisotropies will also be key to constraining other important ICM observables, such as X-ray cavities and jets (driven via AGN feedback; e.g. Gaspari et al. 2020) or the halo structure (shaped by mergers and sloshing; e.g. Ettori et al. 2013b).

Moreover, we cannot exclude the possibility that different elements do not enrich their surroundings with the same efficiency. For instance, specific elements freshly produced by $\mathrm{SNe}$ could be more (or less) easily depleted into dust than others before (or even after) ending up in the ICM. Multi-phase gas, which were not included in the present simulations due to subgrid physics limitations, could also play an important role in this respect. The question of the interplay between hot gas and dust phases of metals (and their enrichment) in the central regions of galactic and cluster hot atmospheres has indeed only just begun to be explored (e.g. Panagoulia et al. 2015; Lakhchaura et al. 2019b; Liu et al. 2019). If this were the case, the observed abundance pattern should be reproduced with comprehensive chemodynamical models rather than linear fits of yield model combinations.

Despite our conservative approach to consider all fits with $\chi_{\text {red }}^{2} \leq 5$ as statistically acceptable, a proper and thorough quantification of all the possible systematic uncertainties (e.g. instrumental response and calibration, spectral code uncertainties, background reproducibility, and subtle residual scatter in the ratios of different systems) that might affect future real observations will be important for the next steps of such studies. In high-redshift clusters, cosmic variance and AGN contamination might also require us to adapt the spatial and spectral analysis. While these systematic errors will contribute to increasing the total uncertainties, dedicated observing strategies (via e.g. larger samples or deeper exposures) will very likely help to compensate for this effect, in addition to optimising the ability of the X-IFU to constrain SN models. Future dedicated work on the total error budget, on the 12 abundance ratios studied here as well as additional ones (Sect. 5.1), will help to refine our results and predict the abilities of distinction between more subtle models.

Finally, the approach adopted in this work relies on simple linear fits using $\chi^{2}$ statistics, allowing comparison with the previous observational studies - using XMM-Newton (e.g. Ettori et al. 2002; de Plaa et al. 2007; Mernier et al. 2016a) and/or Hitomi (Simionescu et al. 2019). Such a methodology will naturally tend to preferentially reproduce ratios that have the smallest error bars. While this is not a problem for the context of this work, better approaches (e.g. using a Bayesian formalism) might be more appropriate for future real data, especially if some specific ratios suffer from additional systematic uncertainties (calibration, spectral codes, etc.) and/or if future nucleosynthesis calculations are provided with formal uncertainties on their 
predicted yields. A thorough, comprehensive comparison between different statistical methods - listing advantages and drawbacks that are relevant for our goals - is left for future work.

\section{Summary and conclusions}

In this paper, which naturally follows Paper I, we use synthetic observations of clusters extracted from hydrodynamical simulations (Rasia et al. 2015) to demonstrate the capabilities of the $\mathrm{X}-\mathrm{IFU}$ in constraining the chemical composition of the ICM. These mock observations were obtained using the X-IFU end-toend simulator SIXTE (Dauser et al. 2019). The measured abundance ratios were then compared to various combinations of existing $\mathrm{AGB}, \mathrm{SN}_{\mathrm{cc}}$, and $\mathrm{SN}_{\mathrm{Ia}}$ yields from the literature. Our main results can be summarised as follows.

1. The $\mathrm{AGB}+\mathrm{SN}_{\mathrm{cc}}+\mathrm{SN}_{\mathrm{Ia}}$ combination of yield models that was used as input in the hydrodynamical simulations (Tornatore et al. 2007) is successfully recovered by the XIFU, both at low $(z=0.1)$ and high $(z=1)$ redshifts. With $\chi_{\text {red }}^{2}=1.49$ for local systems, this combination constitutes the third best fit (out of more than 17000 combinations) with our mock abundance ratios.

2. Complementary to the previous result, we show that a very large number of model combinations $(>99.5 \%)$ could be excluded as they provide a significantly worse fit. Expecting even further improvements when accounting for additional crucial ratios (e.g. $\mathrm{Cr} / \mathrm{Fe}, \mathrm{Mn} / \mathrm{Fe}$ ), this demonstrates that the X-IFU will be able to efficiently favour or rule out specific yield models, therefore providing valuable physical constraints on $\mathrm{AGB}, \mathrm{SN}_{\mathrm{cc}}$, and $\mathrm{SN}_{\mathrm{Ia}}$ and their progenitors. These conclusions are essentially unchanged when the assumption of two co-exisiting $\mathrm{SN}_{\text {Ia }}$ models is considered.

3. For a fixed $\mathrm{AGB}+\mathrm{SN}_{\mathrm{cc}}+\mathrm{SN}_{\mathrm{Ia}}$ model combination (i.e. the one that was recovered as input yields in the simulations), we tried to determine the possible constraints the X-IFU could provide on the stellar IMF, which is a critical parameter to understand the stellar and chemical evolution of the Universe. Through a simple integration of the yields using a Salpeter-like IMF (i.e. power-law like, see Salpeter 1955), we demonstrate that the X-IFU will provide accurate values on the slope (within less than $\sim 12 \%$ ) and the upper mass cutoff of this function. Even further, the Chabrier-like shape of the IMF as used in our input simulations is recovered and favoured in the observations as well. Coupled with other observational evidence, the ability of the X-IFU to pick up a sensible IMF will help to better characterise it and test its (non-)universality.

This study assesses - for the first time - the feasibility of a future instrument in providing constraints on the metal enrichment of the Universe by measuring the chemical composition of the ICM to an unprecedented level. Quite remarkably, most limitations are generally related to our current methodology (which should be considered as a first step) and/or model uncertainties rather than to the X-IFU capabilities. With multiple cluster observations at very high accuracy (which will also help in estimating the total metal budget in the entire cluster volume; e.g. Molendi et al. 2016), and provided that the upcoming XRISM observations will motivate continuous improvements on (i) nucleosynthesis calculations and (ii) spectral atomic codes (as done already with the Hitomi observation of Perseus; Hitomi Collaboration 2018), the X-IFU will provide astronomers with new ways to investigate quantities such as metal production yields in stars, end-of-life models of compact objects, or the mass distribution of stars in the Universe.
Acknowledgements. The authors thank the anonymous referee for useful suggestions that helped to improve this paper, as well as A. Simionescu for insightful discussions. This work is supported by the Lendület LP2016-11 grant awarded by the Hungarian Academy of Sciences. V.B. acknowledges support by the DFG project nr. 415510302. V.B., S.B. and E.R. acknowledge financial contribution from the contract ASI-INAF n.2017-14-H.0. E.R. and S.B. acknowledge the ExaNeSt and Euro Exa projects, funded by the European Union's Horizon 2020 research and innovation programme, under grant agreement No 754337. S.B. also acknowledges support from the INFN INDARK grant. K.D. acknowledges support through ORIGINS, founded by the Deutsche Forschungsgemeinschaft (DFG, German Research Foundation) under Germany's Excellence Strategy - EXC-2094 - 390783311. S.E. acknowledges financial contribution from the contracts ASI-INAF Athena 2015-046-R.0, ASI-INAF Athena 2019-27-HH.0, "Attività di Studio per la comunità scientifica di Astrofisica delle Alte Energie e Fisica Astroparticellare" (Accordo Attuativo ASI-INAF n. 2017 14-H.0), and from INAF "Call per interventi aggiuntivi a sostegno della ricerca di main stream di INAF". M.G. is supported by the Lyman Spitzer Jr. Fellowship (Princeton University) and by NASA Chandra GO8-19104X/GO9-20114X and HST GO-15890.020-A grants. Our French and CNES colleagues are grateful to CNES for their outstanding support in developing the X-IFU for Athena. SRON is supported financially by NWO, the Netherlands Organization for Scientific Research.

\section{References}

Anders, E., \& Grevesse, N. 1989, Geochim. Cosmochim. Acta, 53, 197

Arnaud, K. A. 1996, in Astronomical Data Analysis Software and Systems V, eds. G. H. Jacoby, \& J. Barnes, ASP Conf. Ser., 101, 17

Badenes, C., Borkowski, K. J., Hughes, J. P., Hwang, U., \& Bravo, E. 2006, ApJ, 645, 1373

Barret, D., Lam Trong, T., den Herder, J. W., et al. 2016, in Space Telescopes and Instrumentation 2016: Ultraviolet to Gamma Ray, Proc. SPIE, 9905, 99052F

Barret, D., Lam Trong, T., den Herder, J. W., et al. 2018, in Space Telescopes and Instrumentation 2018: Ultraviolet to Gamma Ray, SPIE Conf. Ser., 10699, $106991 \mathrm{G}$

Bastian, N., Covey, K. R., \& Meyer, M. R. 2010, ARA\&A, 48, 339

Biff, V., Planelles, S., Borgani, S., et al. 2017, MNRAS, 468, 531

Biffi, V., Planelles, S., Borgani, S., et al. 2018a, MNRAS, 476, 2689

Biffi, V., Mernier, F., \& Medvedev, P. 2018b, Space Sci. Rev., 214, 123

Bulbul, E., Smith, R. K., \& Loewenstein, M. 2012, ApJ, 753, 54

Burbidge, E. M., Burbidge, G. R., Fowler, W. A., \& Hoyle, F. 1957, Rev. Mod. Phys., 29, 547

Chabrier, G. 2003, PASP, 115, 763

Clerc, N., Cucchetti, E., Pointecouteau, E., \& Peille, P. 2019, A\&A, 629, A143

Cucchetti, E., Pointecouteau, E., Peille, P., et al. 2018, A\&A, 620, A173

Cucchetti, E., Clerc, N., Pointecouteau, E., Peille, P., \& Pajot, F. 2019, A\&A, 629, A144

Cyburt, R. H., Fields, B. D., Olive, K. A., \& Yeh, T.-H. 2016, Rev. Mod. Phys., 88,015004

Dauser, T., Falkner, S., Lorenz, M., et al. 2019, A\&A, 630, A66

de Grandi, S., \& Molendi, S. 2009, A\&A, 508, 565

De Grandi, S., Eckert, D., Molendi, S., et al. 2016, A\&A, 592, A154

De Masi, C., Vincenzo, F., Matteucci, F., et al. 2019, MNRAS, 483, 2217

de Plaa, J., Werner, N., Bykov, A. M., et al. 2006, A\&A, 452, 397

de Plaa, J., Werner, N., Bleeker, J. A. M., et al. 2007, A\&A, 465, 345

de Plaa, J., Kaastra, J. S., Werner, N., et al. 2017, A\&A, 607, A98

Ettori, S., Fabian, A. C., Allen, S. W., \& Johnstone, R. M. 2002, MNRAS, 331, 635

Ettori, S., Pratt, G. W., de Plaa, J., et al. 2013a, ArXiv e-prints [arXiv:1306.2322]

Ettori, S., Gastaldello, F., Gitti, M., et al. 2013b, A\&A, 555, A93

Ettori, S., Baldi, A., Balestra, I., et al. 2015, A\&A, 578, A46

Ezer, C., Bulbul, E., Nihal Ercan, E., et al. 2017, ApJ, 836, 110

Fabjan, D., Borgani, S., Tornatore, L., et al. 2010, MNRAS, 401, 1670

Fink, M., Kromer, M., Seitenzahl, I. R., et al. 2014, MNRAS, 438, 1762

Gaspari, M., Melioli, C., Brighenti, F., \& D'Ercole, A. 2011, MNRAS, 411, 349

Gaspari, M., Eckert, D., Ettori, S., et al. 2019, ApJ, 884, 169

Gaspari, M., Tombesi, F., \& Cappi, M. 2020, Nat. Astron., 4, 10

Gastaldello, F., \& Molendi, S. 2002, ApJ, 572, 160

Greif, T. H., Glover, S. C. O., Bromm, V., \& Klessen, R. S. 2009, MNRAS, 392 , 1381

Heger, A., \& Woosley, S. E. 2002, ApJ, 567, 532

Heger, A., \& Woosley, S. E. 2010, ApJ, 724, 341

Hillebrandt, W., Kromer, M., Röpke, F. K., \& Ruiter, A. J. 2013, Front. Phys., 8,

116

Hitomi Collaboration (Aharonian, F., et al.) 2017, Nature, 551, 478

Hitomi Collaboration (Aharonian, F., et al.) 2018, PASJ, 70, 12 
Humphrey, P. J., \& Buote, D. A. 2006, ApJ, 639, 136

Iben, I., Jr, \& Renzini, A. 1983, ARA\&A, 21, 271

Iwamoto, K., Brachwitz, F., Nomoto, K., et al. 1999, ApJS, 125, 439

Karakas, A. I. 2010, MNRAS, 403, 1413

Kim, D.-W., Anderson, C., Burke, D., et al. 2019, ApJS, 241, 36

Kirkpatrick, C. C., McNamara, B. R., \& Cavagnolo, K. W. 2011, ApJ, 731, L23

Kromer, M., Fink, M., Stanishev, V., et al. 2013, MNRAS, 429, 2287

Kromer, M., Ohlmann, S. T., Pakmor, R., et al. 2015, MNRAS, 450, 3045

Lakhchaura, K., Truong, N., \& Werner, N. 2019a, MNRAS, 488, L134

Lakhchaura, K., Mernier, F., \& Werner, N. 2019b, A\&A, 623, A17

Leung, S.-C., \& Nomoto, K. 2018, ApJ, 861, 143

Liu, A., Zhai, M., \& Tozzi, P. 2019, MNRAS, 485, 1651

Liu, A., Tozzi, P., Ettori, S., et al. 2020, A\&A, 637, A58

Lodders, K., Palme, H., \& Gail, H.-P. 2009, Landolt Börnstein, 712

Madau, P., \& Dickinson, M. 2014, ARA\&A, 52, 415

Maeda, K., Röpke, F. K., Fink, M., et al. 2010, ApJ, 712, 624

Mantz, A. B., Allen, S. W., Morris, R. G., et al. 2017, MNRAS, 472, 2877

Maoz, D., Mannucci, F., \& Nelemans, G. 2014, ARA\&A, 52, 107

Marquardt, K. S., Sim, S. A., Ruiter, A. J., et al. 2015, A\&A, 580, A118

Matteucci, F., \& Chiappini, C. 2005, PASA, 22, 49

McCarthy, I. G., Schaye, J., Ponman, T. J., et al. 2010, MNRAS, 406, 822

McDonald, M., Bulbul, E., de Haan, T., et al. 2016, ApJ, 826, 124

Mernier, F., de Plaa, J., Lovisari, L., et al. 2015, A\&A, 575, A37

Mernier, F., de Plaa, J., Pinto, C., et al. 2016a, A\&A, 595, A126

Mernier, F., de Plaa, J., Pinto, C., et al. 2016b, A\&A, 592, A157

Mernier, F., de Plaa, J., Kaastra, J. S., et al. 2017, A\&A, 603, A80

Mernier, F., Biffi, V., Yamaguchi, H., et al. 2018a, Space Sci. Rev., 214, 129

Mernier, F., Werner, N., de Plaa, J., et al. 2018b, MNRAS, 480, L95

Million, E. T., Werner, N., Simionescu, A., \& Allen, S. W. 2011, MNRAS, 418 2744

Molendi, S., Eckert, D., De Grandi, S., et al. 2016, A\&A, 586, A32

Nandra, K., Barret, D., Barcons, X., et al. 2013, ArXiv e-prints [arXiv:1306.2307]

Nomoto, K., \& Hashimoto, M. 1988, Phys. Rep., 163, 13

Nomoto, K., Kobayashi, C., \& Tominaga, N. 2013, ARA\&A, 51, 457

Ohlmann, S. T., Kromer, M., Fink, M., et al. 2014, A\&A, 572, A57

Padovani, P., \& Matteucci, F. 1993, ApJ, 416, 26

Pakmor, R., Kromer, M., Röpke, F. K., et al. 2010, Nature, 463, 61

Pakmor, R., Kromer, M., Taubenberger, S., et al. 2012, ApJ, 747, L10

Panagoulia, E. K., Sanders, J. S., \& Fabian, A. C. 2015, MNRAS, 447, 417

Pointecouteau, E., Reiprich, T. H., Adami, C., et al. 2013, ArXiv e-prints [arXiv:1306.2319]
Rasia, E., Borgani, S., Murante, G., et al. 2015, ApJ, 813, L17

Reiprich, T. H., \& Böhringer, H. 2002, ApJ, 567, 716

Romano, D., Karakas, A. I., Tosi, M., \& Matteucci, F. 2010, A\&A, 522, A32

Roncarelli, M., Gaspari, M., Ettori, S., et al. 2018, A\&A, 618, A39

Salpeter, E. E. 1955, ApJ, 121, 161

Schindler, S., \& Diaferio, A. 2008, Space Sci. Rev., 134, 363

Seitenzahl, I. R., Cescutti, G., Röpke, F. K., Ruiter, A. J., \& Pakmor, R. 2013a, A\&A, 559, L5

Seitenzahl, I. R., Ciaraldi-Schoolmann, F., Röpke, F. K., et al. 2013b, MNRAS, 429, 1156

Seitenzahl, I. R., Kromer, M., Ohlmann, S. T., et al. 2016, A\&A, 592, A57

Shen, K. J., Boubert, D., Gänsicke, B. T., et al. 2018, ApJ, 865, 15

Sim, S. A., Röpke, F. K., Hillebrandt, W., et al. 2010, ApJ, 714, L52

Sim, S. A., Fink, M., Kromer, M., et al. 2012, MNRAS, 420, 3003

Simionescu, A., Werner, N., Böhringer, H., et al. 2009, A\&A, 493, 409

Simionescu, A., Nakashima, S., Yamaguchi, H., et al. 2019, MNRAS, 483, 1701

Sukhbold, T., Ertl, T., Woosley, S. E., Brown, J. M., \& Janka, H.-T. 2016, ApJ, 821,38

Takahashi, T., Kokubun, M., Mitsuda, K., et al. 2018, J. Astron. Telescopes Instrum. Syst., 4, 021402

Thielemann, F.-K., Argast, D., Brachwitz, F., et al. 2003, From Twilight to Highlight: The Physics of Supernovae, eds. W. Hillebrandt, \& B. Leibundgut, 331

Tornatore, L., Borgani, S., Dolag, K., \& Matteucci, F. 2007, MNRAS, 382, 1050

Truong, N., Rasia, E., Biffi, V., et al. 2019, MNRAS, 484, 2896

Urban, O., Werner, N., Allen, S. W., Simionescu, A., \& Mantz, A. 2017, MNRAS, 470, 4583

Urdampilleta, I., Mernier, F., Kaastra, J. S., et al. 2019, A\&A, 629, A31

Utrobin, V. P., Wongwathanarat, A., Janka, H.-T., \& Müller, E. 2015, A\&A, 581, A40

van Dokkum, P. G. 2008, ApJ, 674, 29

Waldman, R., Sauer, D., Livne, E., et al. 2011, ApJ, 738, 21

Wang, F. Y., \& Dai, Z. G. 2011, ApJ, 727, L34

Webbink, R. F. 1984, ApJ, 277, 355

Werner, N., de Plaa, J., Kaastra, J. S., et al. 2006, A\&A, 449, 475

Werner, N., Durret, F., Ohashi, T., Schindler, S., \& Wiersma, R. P. C. 2008, Space Sci. Rev., 134, 337

Werner, N., Urban, O., Simionescu, A., \& Allen, S. W. 2013, Nature, 502, 656

Whelan, J., \& Iben, I., Jr 1973, ApJ, 186, 1007

Woosley, S. E., \& Weaver, T. A. 1995, ApJS, 101, 181

XRISM Science Team 2020, ArXiv e-prints [arXiv:2003.04962] 


\section{Appendix A: List of nucleosynthesis yield models}

Table A.1. AGB yield models used in this work via the abunfit package (see below for references).

\begin{tabular}{lccc}
\hline \hline Category & Name & Ref. & Remarks \\
\hline Input & K10_0.0001 & $\alpha$ & $Z_{\text {init }}=\mathbf{0 . 0 0 5} Z_{\odot}, M \in[0.9,6] M_{\odot}$ \\
& K10_0.004 & $\alpha$ & $Z_{\text {init }}=\mathbf{0 . 2} Z_{\odot}, M \in[\mathbf{0 . 9}, 6] M_{\odot}$ \\
& K10_0.008 & $\alpha$ & $Z_{\text {init }}=\mathbf{0 . 4} Z_{\odot}, M \in[\mathbf{0 . 9}, 6] M_{\odot}$ \\
& K10_0.02 & $\alpha$ & $Z_{\text {init }}=\mathbf{1} Z_{\odot}, M \in[\mathbf{0 . 9}, 6] M_{\odot}$ \\
\hline
\end{tabular}

Notes. All these models (bold) are used in our input cosmological simulations.

Table A.2. $\mathrm{SN}_{\mathrm{cc}}$ yield models used in this work via the abunfit package (see below for references).

\begin{tabular}{|c|c|c|c|}
\hline Category & Name & Ref. & Remarks \\
\hline \multicolumn{4}{|r|}{$\mathrm{SN}_{\mathrm{cc}}$} \\
\hline Input & $\begin{array}{l}\text { Ro10_0 } \\
\text { Ro10_2E-6 } \\
\text { Ro10_2E-4 } \\
\text { Ro10_2E-3 } \\
\text { Ro10_2E-2 }\end{array}$ & $\begin{array}{l}\text { a,b } \\
\text { a,b } \\
\text { a,b } \\
\text { a,b } \\
\text { a,b }\end{array}$ & $\begin{array}{l}Z_{\text {init }}=0 Z_{\odot}, M \in[8,50] M_{\odot} \\
Z_{\text {init }}=0.0001 Z_{\odot}, M \in[8,50] M_{\odot} \\
Z_{\text {init }}=0.01 Z_{\odot}, M \in[8,50] M_{\odot} \\
Z_{\text {init }}=0.1 Z_{\odot}, M \in[8,50] M_{\odot} \\
Z_{\text {init }}=1 Z_{\odot}, M \in[8,50] M_{\odot}\end{array}$ \\
\hline Nomoto & 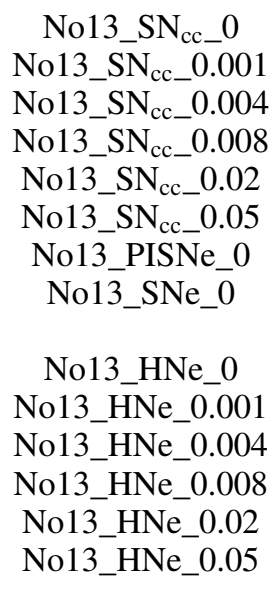 & $\begin{array}{l}\mathrm{c} \\
\mathrm{c} \\
\mathrm{c} \\
\mathrm{c} \\
\mathrm{c} \\
\mathrm{c} \\
\mathrm{c} \\
\mathrm{c}\end{array}$ & $\begin{array}{l}\text { Core-collapse } Z_{\text {init }}=0 Z_{\odot}, M \in[11,140] M_{\odot} \\
\text { Core-collapse } Z_{\text {init }}=0.05 Z_{\odot}, M \in[11,40] M_{\odot} \\
\text { Core-collapse } Z_{\text {init }}=0.2 Z_{\odot}, M \in[11,40] M_{\odot} \\
\text { Core-collapse } Z_{\text {init }}=0.4 Z_{\odot}, M \in[11,40] M_{\odot} \\
\text { Core-collapse } Z_{\text {init }}=1 Z_{\odot}, M \in[11,40] M_{\odot} \\
\text { Core-collapse } Z_{\text {init }}=2.5 Z_{\odot}, M \in[11,40] M_{\odot} \\
\text { Pair-instability } \mathrm{SN}_{e} Z_{\text {init }}=0 Z_{\odot}, M \in[140,300] M_{\odot} \\
\text { Core-collapse } M \in[11,140] M_{\odot} \text { and pair-instability } M \quad \in \\
{[140,300] M_{\odot}, Z_{\text {init }}=0 Z_{\odot}} \\
\text { Hyper-novae } Z_{\text {init }}=0 Z_{\odot} Z_{\odot}, M \in[20,140] M_{\odot} \\
\text { Hyper-novae } Z_{\text {init }}=0.05 Z_{\odot}, M \in[20,40] M_{\odot} \\
\text { Hyper-novae } Z_{\text {init }}=0.2 Z_{\odot}, M \in[20,40] M_{\odot} \\
\text { Hyper-novae } Z_{\text {init }}=0.4 Z_{\odot}, M \in[20,40] M_{\odot} \\
\text { Hyper-novae } Z_{\text {init }}=1 Z_{\odot}, M \in[20,40] M_{\odot} \\
\text { Hyper-novae } Z_{\text {init }}=2.5 Z_{\odot}, M \in[20,40] M_{\odot}\end{array}$ \\
\hline Massive & $\begin{array}{c}\text { He0210_SN }{ }_{\text {cc_0 }} \\
\text { He0210_PISNe_0 } \\
\text { He0210_SNe_0 }\end{array}$ & $\begin{array}{l}\mathrm{d}, \mathrm{e} \\
\mathrm{d}, \mathrm{e} \\
\mathrm{d}, \mathrm{e}\end{array}$ & $\begin{array}{l}\text { Core-collapse } Z_{\text {init }}=0 Z_{\odot}, M \in[10,100] M_{\odot} \\
\text { Pair-instability } \mathrm{SN}_{e} Z_{\text {init }}=0 Z_{\odot}, M \in[140,260] M_{\odot} \\
\text { Core-collapse } M \in[10,100] M_{\odot} \text { and pair-instability, } M \in \\
{[140,260] M_{\odot}, Z_{\text {init }}=0 Z_{\odot}}\end{array}$ \\
\hline Neutrino & Su16_N20 & $\mathrm{f}$ & $\begin{array}{l}\text { Incl. neutrino transport, calibrated for a Nomoto \& Hashimoto } \\
(1988) \text { progenitor to explode as SN1987A, } Z_{\text {init }}=1 Z_{\odot}, M \in \\
{[12,120] M_{\odot}} \\
\text { Incl. neutrino transport, calibrated for a Utrobin et al. (2015) pro- } \\
\text { genitor to explode as SN1987A, } Z_{\text {init }}=1 Z_{\odot}, M \in[12,120] M_{\odot}\end{array}$ \\
\hline
\end{tabular}

Notes. Models used in our input cosmological simulations are given in bold. 
F. Mernier et al.: Constraining the origin and models of chemical enrichment in galaxy clusters using the Athena X-IFU

Table A.3. $\mathrm{SN}_{\text {Ia }}$ yield models used in this work via the abunfit package (continues on next pages, see below for references).

\begin{tabular}{|c|c|c|c|}
\hline Category & Name & Ref. & Remarks \\
\hline \multicolumn{4}{|r|}{$\mathrm{SN}_{\mathrm{Ia}}$} \\
\hline Input & Th03 & 1 & $1 D$ deflagration \\
\hline \multirow[t]{6}{*}{ Bravo } & DDTa & 2 & 1D delayed-detonation, fits the Tycho SNR, $\rho_{T, 7}=3.9$ \\
\hline & DDTb & 2 & (priv. comm., does not fit Tycho SNR, unpublished) \\
\hline & DDTc & 2 & 1D delayed-detonation, fits the Tycho SNR, $\rho_{T, 7}=2.2$ \\
\hline & DDTd & 2 & (priv. comm., does not fit Tycho SNR, unpublished) \\
\hline & DDTe & 2 & 1D delayed-detonation, fits the Tycho SNR, $\rho_{T, 7}=1.3$ \\
\hline & DDTf & 2 & (priv. comm., does not fit Tycho SNR, unpublished) \\
\hline \multirow[t]{8}{*}{ Ca-rich gap } & CO.45HE. 2 & 3 & Ca-rich SNe, $M_{\mathrm{CO}}=0.45, M_{\mathrm{He}}=0.2$ \\
\hline & CO.55HE. 2 & 3 & Ca-rich SNe, $M_{\mathrm{CO}}=0.55, M_{\mathrm{He}}=0.2$ \\
\hline & CO.5HE. 15 & 3 & Ca-rich SNe, $M_{\mathrm{CO}}=0.50, M_{\mathrm{He}}=0.15$ \\
\hline & CO.5HE. 2 & 3 & Ca-rich SNe, $M_{\mathrm{CO}}=0.50, M_{\mathrm{He}}=0.2$ \\
\hline & CO.5HE. 2 C. 3 & 3 & $\begin{array}{l}\text { Ca-rich SNe, } M_{\mathrm{CO}}=0.50, M_{\mathrm{He}}=0.2,30 \% \text { mixing He-core } \\
\text { layer }\end{array}$ \\
\hline & CO.5HE. 2 N.02 & 3 & Ca-rich SNe, $M_{\mathrm{CO}}=0.50, M_{\mathrm{He}}=0.2,2 \% \mathrm{~N}$ in He layer \\
\hline & CO.5HE. 3 & 3 & Ca-rich SNe, $M_{\mathrm{CO}}=0.50, M_{\mathrm{He}}=0.3$ \\
\hline & CO.6HE. 2 & 3 & Ca-rich SNe, $M_{\mathrm{CO}}=0.60, M_{\mathrm{He}}=0.2$ \\
\hline \multirow[t]{3}{*}{$2 \mathrm{D}$} & C-DEF & 4 & 2D deflagration $\rho_{9}=2.9$ \\
\hline & C-DDT & 4 & 2D delayed-detonation $\rho_{9}=2.9, \rho_{T, 7}=1.0$ \\
\hline & O-DDT & 4 & $\begin{array}{l}2 \mathrm{D} \text { delayed-detonation } \rho_{9}=2.9, \rho_{T, 7}=1.0 \text {, off-center igni- } \\
\text { tion }\end{array}$ \\
\hline \multirow[t]{14}{*}{$3 \mathrm{D}$} & N1def & 5 & 3D deflagration $\rho_{9}=2.9,1$ ignition spot \\
\hline & N3def & 5 & $3 \mathrm{D}$ deflagration $\rho_{9}=2.9,3$ ignition spots \\
\hline & N5def & 5 & 3D deflagration $\rho_{9}=2.9,5$ ignition spots \\
\hline & N10def & 5 & $3 \mathrm{D}$ deflagration $\rho_{9}=2.9,10$ ignition spots \\
\hline & N20def & 5 & 3D deflagration $\rho_{9}=2.9,20$ ignition spots \\
\hline & N40def & 5 & 3D deflagration $\rho_{9}=2.9,40$ ignition spots \\
\hline & N100Hdef & 5 & 3D deflagration $\rho_{9}=1.0,100$ ignition spots \\
\hline & N100def & 5 & $3 \mathrm{D}$ deflagration $\rho_{9}=2.9,100$ ignition spots \\
\hline & N100Ldef & 5 & $3 \mathrm{D}$ deflagration $\rho_{9}=5.5,100$ ignition spots \\
\hline & N150def & 5 & 3D deflagration $\rho_{9}=2.9,150$ ignition spots \\
\hline & N200def & 5 & $3 \mathrm{D}$ deflagration $\rho_{9}=2.9,200$ ignition spots \\
\hline & N300Cdef & 5 & 3D deflagration $\rho_{9}=2.9,300$ centred ignition spots \\
\hline & N1600def & 5 & 3D deflagration $\rho_{9}=2.9,1600$ ignition spots \\
\hline & N1600Cdef & 5 & 3D deflagration $\rho_{9}=2.9,1600$ centred ignition spots \\
\hline \multirow[t]{12}{*}{$3 \mathrm{D}$} & $\mathrm{N} 1$ & 6 & 3D delayed-detonation $\rho_{9}=2.9,1$ ignition spot \\
\hline & N3 & 6 & 3D delayed-detonation $\rho_{9}=2.9,3$ ignition spots \\
\hline & N5 & 6 & $3 \mathrm{D}$ delayed-detonation $\rho_{9}=2.9,5$ ignition spots \\
\hline & N10 & 6 & 3D delayed-detonation $\rho_{9}=2.9,10$ ignition spots \\
\hline & N20 & 6 & 3D delayed-detonation $\rho_{9}=2.9,20$ ignition spots \\
\hline & N40 & 6 & 3D delayed-detonation $\rho_{9}=2.9,40$ ignition spots \\
\hline & $\mathrm{N} 100 \mathrm{H}$ & 6 & $3 \mathrm{D}$ delayed-detonation $\rho_{9}=1.0,100$ ignition spots \\
\hline & N100 & 6 & $3 \mathrm{D}$ delayed-detonation $\rho_{9}=2.9,100$ ignition spots \\
\hline & N100L & 6 & 3D delayed-detonation $\rho_{9}=5.5,100$ ignition spots \\
\hline & $\mathrm{N} 150$ & 6 & 3D delayed-detonation $\rho_{9}=2.9,150$ ignition spots \\
\hline & N200 & 6 & $3 \mathrm{D}$ delayed-detonation $\rho_{9}=2.9,200$ ignition spots \\
\hline & $\mathrm{N} 300 \mathrm{C}$ & 6 & 3D delayed-detonation $\rho_{9}=2.9,300$ centred ignition spots \\
\hline
\end{tabular}

Notes. The model used in our input cosmological simulations is given in bold.

References. SN $\mathrm{I}_{\mathrm{Ia}}$ : (1) Thielemann et al. (2003); (2) Badenes et al. (2006); (3) Waldman et al. (2011); (4) Maeda et al. (2010); (5) Fink et al. (2014); (6) Seitenzahl et al. (2013b); (7) Ohlmann et al. (2014); (8) Kromer et al. (2015); (9) Seitenzahl et al. (2016); (10) Sim et al. (2012); (11) Marquardt et al. (2015); (12) Leung \& Nomoto (2018); (13) Sim et al. (2010); (14) Shen et al. (2018); (15) Pakmor et al. (2010); (16) Pakmor et al.

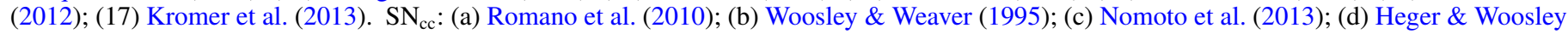
(2002); (e) Heger \& Woosley (2010); (f) Sukhbold et al. (2016). AGB: $(\alpha)$ Karakas (2010). 
Table A.3. continued.

\begin{tabular}{|c|c|c|c|}
\hline Category & Name & Ref. & Remarks \\
\hline & N1600 & 6 & 3D delayed-detonation $\rho_{9}=2.9,1600$ ignition spots \\
\hline & N1600C & 6 & 3D delayed-detonation $\rho_{9}=2.9,1600$ centred ignition spots \\
\hline & N100_Z0.5 & 6 & $\begin{array}{l}\text { 3D delayed-detonation } \rho_{9}=2.9,100 \text { ignition spots, } Z_{\text {init }}= \\
0.5 Z_{\odot}\end{array}$ \\
\hline & N100_Z0.1 & 6 & $\begin{array}{l}\text { 3D delayed-detonation } \rho_{9}=2.9,100 \text { ignition spots, } Z_{\text {init }}= \\
0.1 Z_{\odot}\end{array}$ \\
\hline & N100_Z0.01 & 6 & $\begin{array}{l}\text { 3D delayed-detonation } \rho_{9}=2.9,100 \text { ignition spots, } Z_{\text {init }}= \\
0.01 Z_{\odot}\end{array}$ \\
\hline \multirow[t]{4}{*}{$3 \mathrm{D}$} & N100_c50 & 7 & N100 with WD homogeneous core with $50 \%$ C (mass) \\
\hline & N100_rpc20 & 7 & N100 with WD C-depleted core with $20 \%$ C (mass) \\
\hline & N100_rpc32 & 7 & N100 with WD C-depleted core with $32 \%$ C (mass) \\
\hline & N100_rpc40 & 7 & N100 with WD C-depleted core with $40 \%$ C (mass) \\
\hline \multirow[t]{25}{*}{ LN18 } & $050-1-c 3-1 P$ & 12 & $2 \mathrm{D}$ deflagr., $\rho_{9}=0.5$, centred ignition, $Z_{\text {init }}=1 Z_{\odot}$ \\
\hline & $100-1-c 3-1 P$ & 12 & $2 \mathrm{D}$ deflagr., $\rho_{9}=1.0$, centred ignit., $Z_{\text {init }}=1 Z_{\odot}$ \\
\hline & $100-0-c 3$ & 12 & $2 \mathrm{D}$ del.-det., $\rho_{9}=1.0$, centred ignit., $Z_{\text {init }}=0 Z_{\odot}$ \\
\hline & $100-0.1-c 3$ & 12 & $2 \mathrm{D}$ del.-det., $\rho_{9}=1.0$, centred ignit., $Z_{\text {init }}=0.1 Z_{\odot}$ \\
\hline & $100-0.5-c 3$ & 12 & $2 \mathrm{D}$ del.-det., $\rho_{9}=1.0$, centred ignit., $Z_{\text {init }}=0.5 Z_{\odot}$ \\
\hline & $100-1-c 3$ & 12 & $2 \mathrm{D}$ del.-det., $\rho_{9}=1.0$, centred ignit., $Z_{\text {init }}=1 Z_{\odot}$ \\
\hline & $100-2-c 3$ & 12 & $2 \mathrm{D}$ del.-det., $\rho_{9}=1.0$, centred ignit., $Z_{\text {init }}=2 Z_{\odot}$ \\
\hline & $100-3-c 3$ & 12 & $2 \mathrm{D}$ del.-det., $\rho_{9}=1.0$, centred ignit., $Z_{\text {init }}=3 Z_{\odot}$ \\
\hline & $100-5-c 3$ & 12 & $2 \mathrm{D}$ del.-det., $\rho_{9}=1.0$, centred ignit., $Z_{\text {init }}=5 Z_{\odot}$ \\
\hline & $300-1-c 3-1 P$ & 12 & $2 \mathrm{D}$ deflagr., $\rho_{9}=3.0$, centred ignit., $Z_{\text {init }}=1 Z_{\odot}$ \\
\hline & $300-0-\mathrm{c} 3$ & 12 & $2 \mathrm{D}$ del.-det., $\rho_{9}=3.0$, centred ignit., $Z_{\text {init }}=0 Z_{\odot}$ \\
\hline & $300-0.1-c 3$ & 12 & $2 \mathrm{D}$ del.-det., $\rho_{9}=3.0$, centred ignit., $Z_{\text {init }}=0.1 Z_{\odot}$ \\
\hline & $300-0.5-c 3$ & 12 & $2 \mathrm{D}$ del.-det., $\rho_{9}=3.0$, centred ignit., $Z_{\text {init }}=0.5 Z_{\odot}$ \\
\hline & $300-1-c 3$ & 12 & $2 \mathrm{D}$ del.-det., $\rho_{9}=3.0$, centred ignit., $Z_{\text {init }}=1 Z_{\odot}$ \\
\hline & $300-2-c 3$ & 12 & $2 \mathrm{D}$ del.-det., $\rho_{9}=3.0$, centred ignit., $Z_{\text {init }}=2 Z_{\odot}$ \\
\hline & $300-3-c 3$ & 12 & $2 \mathrm{D}$ del.-det., $\rho_{9}=3.0$, centred ignit., $Z_{\text {init }}=3 Z_{\odot}$ \\
\hline & $300-5-c 3$ & 12 & 2D del.-det., $\rho_{9}=3.0$, centred ignit., $Z_{\text {init }}=5 Z_{\odot}$ \\
\hline & $500-1-c 3-1 P$ & 12 & $2 \mathrm{D}$ deflagr., $\rho_{9}=5.0$, centred ignit., $Z_{\text {init }}=1 Z_{\odot}$ \\
\hline & $500-0-c 3$ & 12 & $2 \mathrm{D}$ del.-det., $\rho_{9}=5.0$, centred ignit., $Z_{\text {init }}=0 Z_{\odot}$ \\
\hline & $500-0.1-c 3$ & 12 & $2 \mathrm{D}$ del.-det., $\rho_{9}=5.0$, centred ignit., $Z_{\text {init }}=0.1 Z_{\odot}$ \\
\hline & $500-0.5-c 3$ & 12 & $2 \mathrm{D}$ del.-det., $\rho_{9}=5.0$, centred ignit., $Z_{\text {init }}=0.5 Z_{\odot}$ \\
\hline & $500-1-c 3$ & 12 & $2 \mathrm{D}$ del.-det., $\rho_{9}=5.0$, centred ignit., $Z_{\text {init }}=1 Z_{\odot}$ \\
\hline & $500-2-c 3$ & 12 & $2 \mathrm{D}$ del.-det., $\rho_{9}=5.0$, centred ignit., $Z_{\text {init }}=2 Z_{\odot}$ \\
\hline & $500-3-c 3$ & 12 & $2 \mathrm{D}$ del.-det., $\rho_{9}=5.0$, centred ignit., $Z_{\text {init }}=3 Z_{\odot}$ \\
\hline & $500-5-c 3$ & 12 & $2 \mathrm{D}$ del.-det., $\rho_{9}=5.0$, centred ignit., $Z_{\text {init }}=5 Z_{\odot}$ \\
\hline HWD & N5_hy & 8 & Hybrid WD (CO and ONe layers) \\
\hline GCD & GCD200 & 9 & Gravity-confined detonation \\
\hline \multirow[t]{6}{*}{ DbleDet } & CSDD-L & 10 & 2D converging-shock double-deton., $M_{\mathrm{CO}}=0.45 M_{\odot}$ \\
\hline & CSDD-S & 10 & $2 \mathrm{D}$ converging-shock double-deton., $M_{\mathrm{CO}}=0.58 M_{\odot}$ \\
\hline & ELDD-L & 10 & $2 \mathrm{D}$ edge-lit double-detonation, $M_{\mathrm{CO}}=0.45 M_{\odot}$ \\
\hline & ELDD-S & 10 & 2D edge-lit double-detonation, $M_{\mathrm{CO}}=0.58 M_{\odot}$ \\
\hline & HeD-L & 10 & 2D He detonation only, $M_{\mathrm{CO}}=0.45 M_{\odot}$ \\
\hline & HeD-S & 10 & 2D He detonation only, $M_{\mathrm{CO}}=0.58 M_{\odot}$ \\
\hline
\end{tabular}


Table A.3. continued.

\begin{tabular}{|c|c|c|c|}
\hline Category & Name & Ref. & Remarks \\
\hline $\mathrm{ONe}$ & $\begin{array}{l}\text { CO15e7 } \\
\text { ONe10e7 } \\
\text { ONe13e7 } \\
\text { ONe15e7 } \\
\text { ONe17e7 } \\
\text { ONe20e7 }\end{array}$ & $\begin{array}{l}11 \\
11 \\
11 \\
11 \\
11 \\
11\end{array}$ & $\begin{array}{l}\text { 2D }\left(\text { sub- } M_{\mathrm{Ch}}\right) \text { detonation carbon-oxygen WD, } \rho_{9}=0.15 \\
\text { 2D }\left(\text { sub- } M_{\mathrm{Ch}}\right) \text { detonation oxygen-neon WD, } \rho_{9}=0.10 \\
\text { 2D }\left(\text { sub- } M_{\mathrm{Ch}}\right) \text { detonation oxygen-neon WD, } \rho_{9}=0.13 \\
\text { 2D }\left(\text { sub- } M_{\mathrm{Ch}}\right) \text { detonation oxygen-neon WD, } \rho_{9}=0.15 \\
\text { 2D }\left(\text { sub- } M_{\mathrm{Ch}}\right) \text { detonation oxygen-neon WD, } \rho_{9}=0.17 \\
\left.\text { 2D (sub- } M_{\mathrm{Ch}}\right) \text { detonation oxygen-neon WD, } \rho_{9}=0.20\end{array}$ \\
\hline Det & $\begin{array}{l}\text { det_0.81 } \\
\text { det_0.88 } \\
\text { det_0.97 } \\
\text { det_1.06 } \\
\text { det_1.15 } \\
\text { det_1.06_0.075Ne }\end{array}$ & $\begin{array}{l}13 \\
13 \\
13 \\
13 \\
13 \\
13\end{array}$ & $\begin{array}{l}1 \mathrm{D}\left(\text { sub- } M_{\mathrm{Ch}}\right) \text { pure detonation, } M_{\mathrm{CO}}=0.81 M_{\odot}, \rho_{7}=1.0 \\
1 \mathrm{D}\left(\text { sub- } M_{\mathrm{Ch}}\right) \text { pure detonation, } M_{\mathrm{CO}}=0.88 M_{\odot}, \rho_{7}=1.45 \\
1 \mathrm{D}\left(\text { sub- } M_{\mathrm{Ch}}\right) \text { pure detonation, } M_{\mathrm{CO}}=0.97 M_{\odot}, \rho_{7}=2.4 \\
1 \mathrm{D}\left(\text { sub- } M_{\mathrm{Ch}}\right) \text { pure detonation, } M_{\mathrm{CO}}=1.06 M_{\odot}, \rho_{7}=4.15 \\
1 \mathrm{D}\left(\text { sub- } M_{\mathrm{Ch}}\right) \text { pure detonation, } M_{\mathrm{CO}}=1.15 M_{\odot}, \rho_{7}=7.9 \\
1 \mathrm{D}\left(\text { sub- } M_{\mathrm{Ch}}\right) \text { pure detonation, } M_{\mathrm{CO}}=1.06 M_{\odot}, \rho_{7}=4.15, \\
\mathrm{C} / \mathrm{O} / \mathrm{Ne} \text { mass fraction }=0.425 / 0.5 / 0.075\end{array}$ \\
\hline $6 \mathrm{D}$ & Sh18_Ma_b_Zc_d & 14 & $\begin{array}{l}\left.\text { 3D (sub- } M_{\mathrm{Ch}}\right) \text { dynamically-driven double-degenerate dou- } \\
\text { ble detonation, } 159 \text { models in total for different WD } \\
\text { masses }\left(\mathrm{Ma} \in\{0.8,0.85,0.9,1.0,1.1\} M_{\odot}\right) \text {, } \mathrm{C} / \mathrm{O} \text { compo- } \\
\text { sitions }(\mathrm{b} \in\{30 / 70,50 / 50\}) \text {, metallicity } Z_{\text {init }}(\mathrm{Zc} \in \\
\{0,0.005,0.01,0.02\}) \text {, and normalizations of the }{ }^{12} \mathrm{C}+{ }^{16} \mathrm{O} \\
\text { reaction rate }(\mathrm{d} \in\{0.1,1.0\})\end{array}$ \\
\hline Merger & $\begin{array}{l}09 \_09 \\
11 \_09\end{array}$ & 16 & $\begin{array}{l}3 \mathrm{D} \quad\left(\text { sub- } M_{\mathrm{Ch}}\right) \text { violent } \text { WD merger (double-degen.), } \\
0.9+0.9 M_{\odot} \\
\left.3 \mathrm{D} \quad \text { (sub- } M_{\mathrm{Ch}}\right) \text { violent } \mathrm{WD} \text { merger (double-degen.), } \\
1.1+0.9 M_{\odot}\end{array}$ \\
\hline Merger2 & 09_076 & 17 & $\begin{array}{l}3 \mathrm{D} \quad\left(\text { sub- } M_{\mathrm{Ch}}\right) \text { violent } \text { WD merger } \text { (double-degen.), } \\
0.9+0.76 M_{\odot}, Z_{\text {init }}=1 Z_{\odot} \\
\text { 3D }\left(\text { sub- } M_{\mathrm{Ch}}\right) \text { violent } \text { WD merger } \text { (double-degen.), } \\
0.9+0.76 M_{\odot}, Z_{\text {init }}=0.01 Z_{\odot}\end{array}$ \\
\hline
\end{tabular}

